\title{
Phase transitions and molecular dynamics of n-hexadecanol confined in silicon nanochannels
}

\author{
R. Berwanger ${ }^{1}$, A. Henschel ${ }^{2}$, K. Knorr ${ }^{2}$, P. Huber ${ }^{2}$, and R. Pelster ${ }^{1}$ \\ Universität des Saarlandes, ${ }^{1}$ FR 7.2 Experimentalphysik \& ${ }^{2}$ FR 7.3 Technische Physik, \\ 66041 Saarbrücken, Germany
}

\begin{abstract}
We present a combined x-ray diffraction and infrared spectroscopy study on the phase behavior and molecular dynamics of n-hexadecanol in its bulk state and confined in an array of aligned nanochannels of $8 \mathrm{~nm}$ diameter in mesoporous silicon. Under confinement the transition temperatures between the liquid, the rotator $\mathrm{R}_{I I}$ and the crystalline $\mathrm{C}$ phase are lowered by approximately $20 \mathrm{~K}$. While bulk n-hexadecanol exhibits at low temperatures a polycrystalline mixture of orthorhombic $\beta$ - and monoclinic $\gamma$-forms, geometrical confinement favors the more simple $\beta$-form: only crystallites are formed, where the chain axis are parallel to the layer normal. However, the $\gamma$-form, in which the chain axis are tilted with respect to the layer normal, is entirely suppressed. The $\beta$-crystallites form bi-layers, that are not randomly orientated in the pores. The molecules are arranged with their long axis perpendicular to the long channel axis. With regard to the molecular dynamics, we were able to show that confinement does not affect the inner-molecular dynamics of the $\mathrm{CH}_{2}$ scissor vibration and to evaluate the inter-molecular force constants in the $\mathrm{C}$ phase.
\end{abstract}

PACS numbers: $64.70 . \mathrm{Nd}, 61.46 . \mathrm{Hk}, 81.07 .-\mathrm{b}, 61.43 . \mathrm{Gt}$

\section{INTRODUCTION}

The physical properties of condensed matter spatially confined in pores or channels of a few nanometer in diameter can differ markedly from the behavior in the bulk state. In particular, phase transitions can be entirely suppressed or significantly altered in comparison to their bulk counterparts $1,2,3,4$. Also the dynamics of condensed matter confined in mesopores, most prominently in the vicinity of glass transitions $5,6,7,8,9,10,11,12,13,14$, can be affected markedly.

Intimately related to these changes in the phase transition phenomenology the architectural principles of molecular solids can substantially differ in the spatially confined state from the bulk state. This depends, however, sensitively on the complexity of the building blocks. For simple van-der-Waals systems, such as $\mathrm{Ar}$ and $\mathrm{N}_{2}$, a remarkable robustness of the bulk structures has been found for the solid state under confinement $15,16,17$. By contrast, the structural properties of pore fillings built out of more complex building blocks, such as linear hydrocarbons $18,19,20,21,22$ or liquid crystals $\frac{23,24}{2}$ are very susceptible to confinement on the meso- and nanoscale. For example, a quenching of the lamellar ordering of molecular crystals of n-alkanes has been observed in tortuous silica mesopores of Vycor 25 . However, in tubular channels of mesoporous silicon this building principle of hydrocarbon molecular crystals survives, albeit a peculiar texture has been observed for the pore confined solids $\frac{19}{}$ : The long axes of the molecules and thus the stacking direction of the lamellae are oriented perpendicular to the long axis of the pores.

Here we present an experimental study on a mediumlength, linear alcohol $\mathrm{C}_{16} \mathrm{H}_{33} \mathrm{OH}$, a representative of the 1-alcohol series, imbibed in mesoporous silicon. We explore the phase behavior of the confined alcohol by a com- bination of x-ray diffraction and infrared spectroscopy measurements. As we shall demonstrate, we profit in those experiments both from the parallel alignment of the silicon channels and from the transparency of the silicon host in the infrared region.

\section{EXPERIMENTAL}

The porous silicon samples used in this study were prepared by electrochemical etching of a heavily p-doped (100) silicon wafer ${ }^{1}$ with a current density of $13 \frac{\mathrm{mA}}{\mathrm{cm}^{2}}$ in a solution composed of $\mathrm{HF}$, ethanol and $\mathrm{H}_{2} \mathrm{O}(1: 3: 1$ per volume $)^{26,27,28}$. These conditions led to a parallel arrangement of non-interconnected channels oriented with their long axes along the $<100>$ crystallographic direction of silicon, which coincides with the normal of the wafer surface. After the porous layer had reached the desired thickness of 70 microns, the anodization current was increased by a factor of ten with the result that the porous layer was released from the bulk wafer underneath. Using nitrogen sorption isotherms at $T=77 \mathrm{~K}$, we determined a porosity of $60 \%$ and a mean channel diameter of $8 \mathrm{~nm}$. The single crystalline character of the matrix was checked by x-ray diffraction. Transmission electron micrographs of channel cross sections indicate polygonal, rough channel perimeters rather than circular, smooth circumferences 29.

The matrix both for the infrared spectroscopy and the $\mathrm{x}$-ray measurements were filled completely via capillary action (spontaneous imbibition) with liquefied

\footnotetext{
${ }^{1}$ producer: SiMat, Landsberg, Germany; specific conductivity: $\rho=0.01-0.025 \Omega \mathrm{cm}$.
} 
$\mathrm{C}_{16} \mathrm{H}_{33} \mathrm{OH}^{30}$. Bulk excess material at the surface was removed by paper tissues.

Infrared spectra in a range of wavenumbers $\bar{\nu}$ from 4000 to $800 \mathrm{~cm}^{-1}$ with a resolution of $1 \mathrm{~cm}^{-1}$ were measured with a Fourier Transform Spectrometer (FTIR Perkin Elmer System 2000). This range corresponds to frequencies from $3 \cdot 10^{13} \mathrm{~Hz}$ to $1.2 \cdot 10^{14} \mathrm{~Hz}$ (wavelengths from $10 \mu \mathrm{m}$ to $2.5 \mu \mathrm{m}$ ). For both the bulk material and the filled porous samples the same sample holder was used, i. e. a copper cell with two transparent $\mathrm{KBr}$ windows. In the confinement experiments the long channel axes were oriented parallel to the beam axis, i. e. perpendicular to the electric field vector. The sample holder was placed into a cryostat (a closed cycle refrigerator CTI cryogenics, Model 22) allowing us to vary the temperature from 50 to $340 \mathrm{~K}$. The temperature was controlled with a LakeShore 340 temperature controller with a precision of $\pm 0.25 \mathrm{~K}$. All IR-spectra that we show in the following were measured during cooling (typical cooling rates were of the order of $0.5 \mathrm{~K} / \mathrm{min}$ ). Heating scans show the same behavior except for the transition temperatures, which are some degrees higher (see below).

For the x-ray measurements the sample was mounted on a frame in a sample cell consisting of a Peltier cooled base plate and a Be cap. The cell was filled with He gas for better thermal contact. The Be cap sits in a vacuum chamber, the outer jacket of which has Mylar windows allowing the passage of the $\mathrm{x}$-rays over a wide range of scattering angles $\theta$ within the scattering plane (see Fig. 1). But the set-up allowed practically no tilt with respect to the scattering plane. The temperature was controlled by a LakeShore 330 over an accessible range from $245 \mathrm{~K}$ up to $370 \mathrm{~K}$. The measurements were carried out on a twocircle x-ray diffractometer with graphite monochromatized $\mathrm{CuK}_{\alpha}$ radiation emanating from a rotating anode. The porous sheet was mounted perpendicular to the scattering plane. The two angles that could be varied were the detector angle $2 \theta$ and the rotation angle $\omega$ about the normal of the scattering plane. The samples were studied as a function of temperature by performing several $\Phi$-scans. In this paper we concentrate on radial $2 \theta-\omega$ Scans in reflection geometry, i.e. along $\mathrm{q}_{\mathrm{p}}$ with $\Phi=0^{\circ}$, and in transmission geometry, i.e. along $\mathrm{q}_{\mathrm{s}}$ with $\Phi=90^{\circ}$ (see Fig. 11).

\section{A. Structure of bulk n-hexadecanol}

n-Hexadecanol, $\mathrm{C}_{16} \mathrm{H}_{33} \mathrm{OH}$, is an almost rod-like molecule with a length of $22 \AA$ and a width of $4 \AA$. The $\mathrm{C}$-atoms of the backbone are in an all-trans-configuration so that they are located in a plane ${ }^{25}$.

At low temperatures n-alcohols form bi-layered crystals in two possible modifications: the so-called $\gamma$-form, i. e. a monoclinic structure as sketched in Fig. $2\left(C_{2 h}^{6}-\right.$

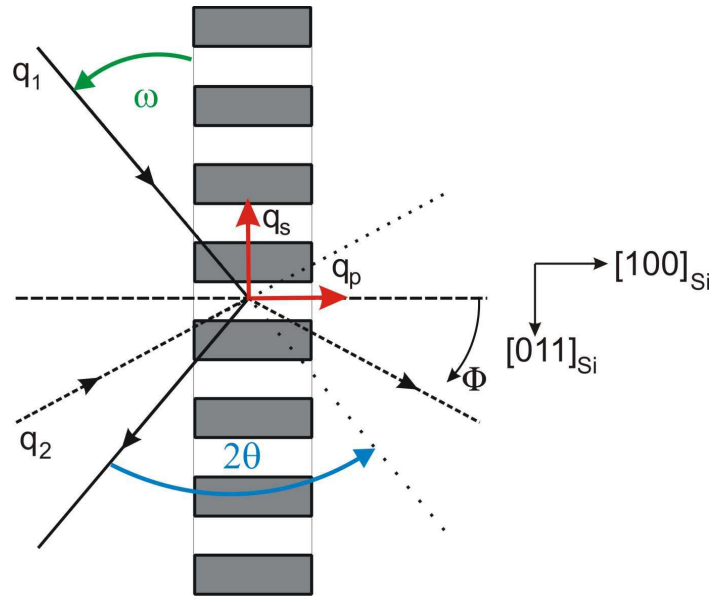

FIG. 1: (Color online) The angular variable $\Phi(\Phi=\omega-\theta$ with $\Phi=0^{\circ}$ for scans along $q_{p}$ and $\Phi=90^{\circ}$ for scans along $\left.q_{\mathrm{s}}\right)$ is indicated. Also shown are the incoming and outgoing x-ray beam for scans along $\mathrm{q}_{\mathrm{p}}$ (solid lines: wave vector component parallel to the pore axis, this means reflection geometry), and along $\mathrm{q}_{\mathrm{s}}$ (dashed lines: wave vector component perpendicular to the pore axis; this is transmission geometry). For scans along $\mathrm{q}_{\mathrm{p}}$, the detector angle $2 \theta$ and the rotation angle $\omega$ are represented.

$\left.A 2 / a^{31.32}\right)$, or the so-called $\beta$-form, i. e. an orthorhombic structure as sketched in Fig. $33^{\beta 3}$. In the $\gamma$-form, the molecules include an angle of $122^{\circ}$ with the layer plane. Within the layers, they are close-packed in a quasi-hexagonal 2D array, described by the rectangular in-plane lattice parameters $a$ and $b$ (according to Ref 32 $a=7.42 \AA$ and $b=4.93 \AA$ holds, so that $a / b=1.5$ ). There are two different alternating orientations for the CC-plane of the backbone leading to a herringbone structure (see Fig. 2b). The $\beta$-form exhibits an identical orientational order of the backbone, but the molecules' axes remain perpendicular to the layers as sketched in Fig. ${ }^{3}{ }^{B 3}$. In addition, gauche- and trans-conformation of the CObond alternate with molecules in this phase, while they are in an all-trans configuration in the $\gamma$-form. In general, the $\gamma$-form dominates at low temperatures for the even alcohols, while the $\beta$-form is more frequent in odd n-alcohols ${ }^{33.34}$. For n-hexadecanol both the orthorhombic $\beta$-form ${ }^{33}$ and the monoclinic $\gamma$-form ${ }^{31,32}$ are reported. Depending on the preparation conditions it is possible to obtain a polycristalline mixture of the monoclinic $\gamma$ - and the orthorhombic $\beta$-form ${ }^{34}$.

Upon heating, the crystalline phase undergoes a transition into a so-called Rotator-(II)-phase $R_{I I}$, which is schematically depicted in Fig. ${ }^{2}{ }^{2}$. This phase has a hexagonal in-plane arrangement with the $c$-direction perpendicular to the cell base. The hexagonal arrangement

\footnotetext{
${ }^{2}$ For several alkanes, there also exists a Rotator-(I)-Phase $R_{I}$, where the molecules switch between two equal positions.
} 

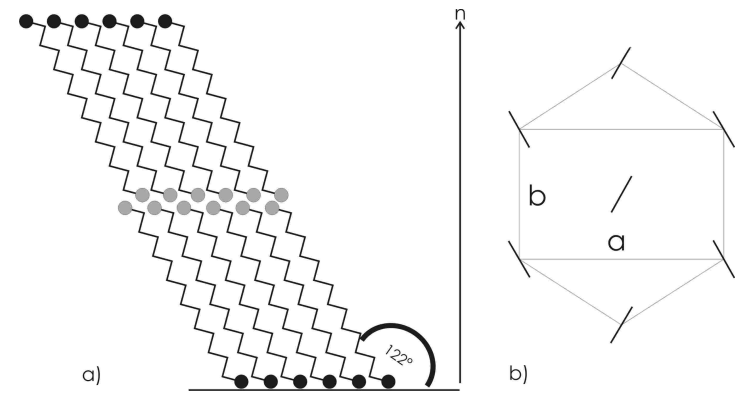

FIG. 2: $\gamma$-form of the crystalline low temperature phase of bulk $\mathrm{C}_{16} \mathrm{H}_{33} \mathrm{OH}(T \leq 310 \mathrm{~K})$. The structure is monoclinic. The left sketch shows the orientation of the molecules with respect to the layer normal $n$, the right sketch the in-plane arrangement, i. e. a projection of the backbones into the a-bplane. Compare with the $\beta$-form sketched in Fig. 3
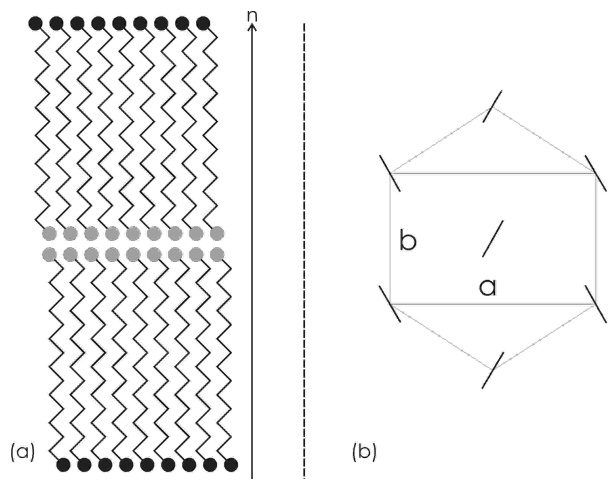

(b)

FIG. 3: $\beta$-form of the crystalline low temperature phase of $\mathrm{C}_{16} \mathrm{H}_{33} \mathrm{OH}$. In contrast to the $\gamma$-form (see Fig. 2 $\mathrm{a}$ ), the long chain axes are not tilted but parallel to the layer normal $n$, i. e. the structure is orthorhombic. Bulk $\mathrm{C}_{16} \mathrm{H}_{33} \mathrm{OH}$ can exhibit a polycrystalline mixture of $\gamma$ - and $\beta$-form (see Sec. IIA). Confinement into nanopores leads to the $\beta$-form (see below, Sec. IIIA).

can be indexed with an orthorhombic cell with a ratio of rectangular basal lattice parameters of $a / b=\sqrt{3} \underline{35}$. On a microscopic level the change in the center of mass lattice from the low-temperature crystalline phase to the rotator phase can be attributed to the onset of hindered rotations of the molecules about their long axes between six equivalent positions (the stars in Fig. 4b). Further heating above $322 \mathrm{~K}$ leads to the liquid state 35 .

\section{RESULTS}

\section{A. Structure of confined n-hexadecanol}

We have determined structures, phase sequences and transition temperatures of $\mathrm{n}-\mathrm{C}_{16} \mathrm{H}_{33} \mathrm{OH}$ confined in mesoporous silicon by x-ray diffractometry. The upper panel in Fig. 5 shows diffraction patterns along $\mathrm{q}_{\mathrm{p}}$ at se-

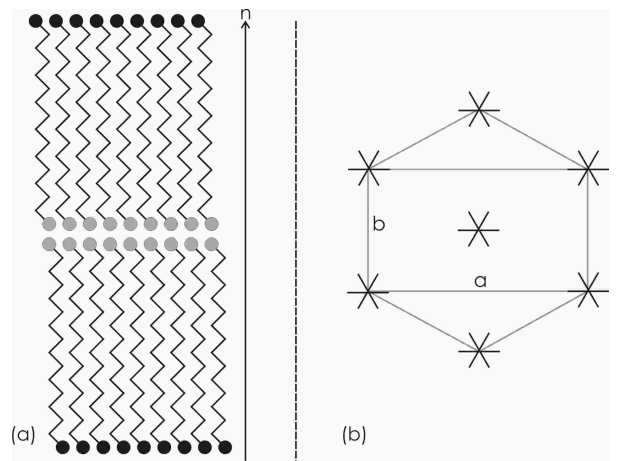

FIG. 4: Structure of bulk n-hexadecanol in the Rotator-(II)phase (for $310 \leq T \leq 322 \mathrm{~K}$ ), a hexagonal arrangement. The right picture shows the perfect hexagonal lattice in the a-bplane. Confined $\mathrm{C}_{16} \mathrm{H}_{33} \mathrm{OH}$ exhibits the same structure in its rotator phase, but in a different temperature range (see below, Table 【).

lected temperatures while cooling. The appearance of a broad Bragg peak at $2 \theta \simeq 21^{\circ}$ indicates solidifaction. Its position is compatible with the leading hexagonal inplane reflection of the $R_{I I}$ phase. Upon further cooling a second peak at $2 \theta \simeq 24^{\circ}$ shows up. This change in the diffraction pattern indicates an uniaxial deformation of the hexagonal lattice. Both reflections can be mapped on a $2 \mathrm{D}$ rectangular mesh characteristic of an uniaxially deformed hexagonal cell. The overall resulting pattern is, however, incompatible with the monoclinic structure of the low temperature bulk crystalline phase.

Additionally to the $\mathrm{q}_{\mathrm{p}}$-scans, we performed also scans for a variety of additional orientations of the scattering vector with regard to the long axis of the channels. These patterns differ markedly, which is indicative of a strong texture of the pore confined cystallized alcohol. It is no powder in the crystallographic sense. In particular, there are strong in-plane reflections and no layering reflections for scans along $\mathrm{q}_{\mathrm{p}}$, while the $\mathrm{q}_{\mathrm{s}}$-scans for the same sample show at least very weak reflections characteristic of a bilayer stacking and only very weak leading in-plane reflections (see Fig. 51). An analysis of the width of the layering reflections yields a coherence length of $7( \pm 1.5) \mathrm{nm}$.

As discussed in more detail in Refs $\frac{19}{}$ and $\frac{36}{}$, the overall picture which emerges from these results can be summed up as follows: the alcohol molecules form orthorhombic structures with a bilayer-stacking direction along the $c$-direction. Within the bilayers (the a-b-plane), the molecules' backbones are untilted with regard to the stacking direction and the backbones are orientationally either fully ordered (in a herringbone fashion) or partially ordered, as known from the $\mathrm{R}_{I}$ phase of n-alkanes. The superlattice reflection characteristic of the full, herringbone type orientational ordering has been searched for and could weakly be detected at low temperatures. The degree of uniaxial deformation of the hexagonal center of mass cell, quantified by the deviation of the ratio $a / b$ from its value in the hexagonal phase $(\sqrt{3})$, also in- 
dicates a full orientational ordered state (see Table $\mathrm{I}, \stackrel{32}{ }$ ). Thus, the diffraction data are compatible with the bulk $\beta$ modification discussed above. This conclusion is also supported by an analysis of the infrared spectroscopy data sets presented below.

More importantly, the peculiar dependency of the diffraction patterns on the orientation of the q-vector with regard to the silicon host indicate that the bi-layer stacking direction is perpendicular to the long axis of the channels and, consequently, that the long axis of the molecules is oriented perpendicular to the long axis of the channels (see Fig. 6). At first glance, this finding may appear somewhat counter-intuitive. Albeit it can be understood as resulting from the crystallization process in a strongly anistropic, capillary-like confined liquid 19,37 . It is a well established principle in single crystal growth that in narrow capillaries the fastest growing crystallization direction prevails over other directions and propagates along the long axes of capillaries ${ }^{38}$. For layered molecular crystals of rod-like building blocks this direction is an in-plane direction, which is perpendicular to the long axis of the rods. If this direction is aligned parallel to the silicon nanochannels due to the crystallization process, it dictates a perpendicular arrangement of the molecules' long axes with regard to the long channel axis, in agreement with the diffraction results presented here.

\begin{tabular}{|c||c|c|c|}
\hline \multicolumn{1}{|c||}{} & bulk & \multicolumn{2}{c|}{ confinement } \\
\cline { 2 - 4 } & cryst. & $\mathrm{R}_{I I}$ & cryst. \\
\hline \hline $\mathrm{a}[\AA]$ & 7.42 & 8.35 & 7.33 \\
\hline $\mathrm{b}[\AA]$ & 4.93 & 4.82 & 5.04 \\
\hline $\mathrm{a} / \mathrm{b}$ & 1.51 & $\sqrt{3}$ & 1.45 \\
\hline $\mathrm{d}[\AA]$ & 8.91 & 9.64 & 8.90 \\
\hline
\end{tabular}

TABLE I: Lattice parameters a and b of bulk and confined $\mathrm{C}_{16} \mathrm{H}_{33} \mathrm{OH}$ and the diagonal $d=\sqrt{a^{2}+b^{2}}$ of the subcell (see Fig. 14). The confined data result from our x-ray measurements and the bulk data are taken from the literature ${ }^{32}$.

The temperature dependent diffraction study allows us to gain additional information on the relative stability of the different nanochannel confined phases. In Table I we display the phase transition temperatures of confined $\mathrm{C}_{16} \mathrm{H}_{33} \mathrm{OH}$ as inferred from the appearance or disappaerance of characteristic Bragg peaks. There is a hysteresis between heating and cooling for both the fluid- $\mathrm{R}_{I I^{-}}$and the $\mathrm{R}_{I I}$-C-transition ( $8 \mathrm{~K}$ and $3 \mathrm{~K}$, respectively). Compared to the bulk data (see also Tab. III), the transition temperatures of pore confined $\mathrm{C}_{16} \mathrm{H}_{33} \mathrm{OH}$ are lowered. On cooling, the lowering is of the order of $\Delta T=18 \mathrm{~K}$ for the fluid- $R_{I I^{-}}$transition and $\Delta T=26 \mathrm{~K}$ for the $R_{I I}$-Ctransition. This observation is analogous to phase transitions shifts reported for other pore condensates 2.3 .

Furthermore, the temperature range of the confined $R_{I I}$ phase, $14 \mathrm{~K}$ upon cooling and $19 \mathrm{~K}$ upon heating, is larger than that of the bulk material $(12 \mathrm{~K})$. Obviously, confinement stabilizes the orientational disordered $R_{I I}$
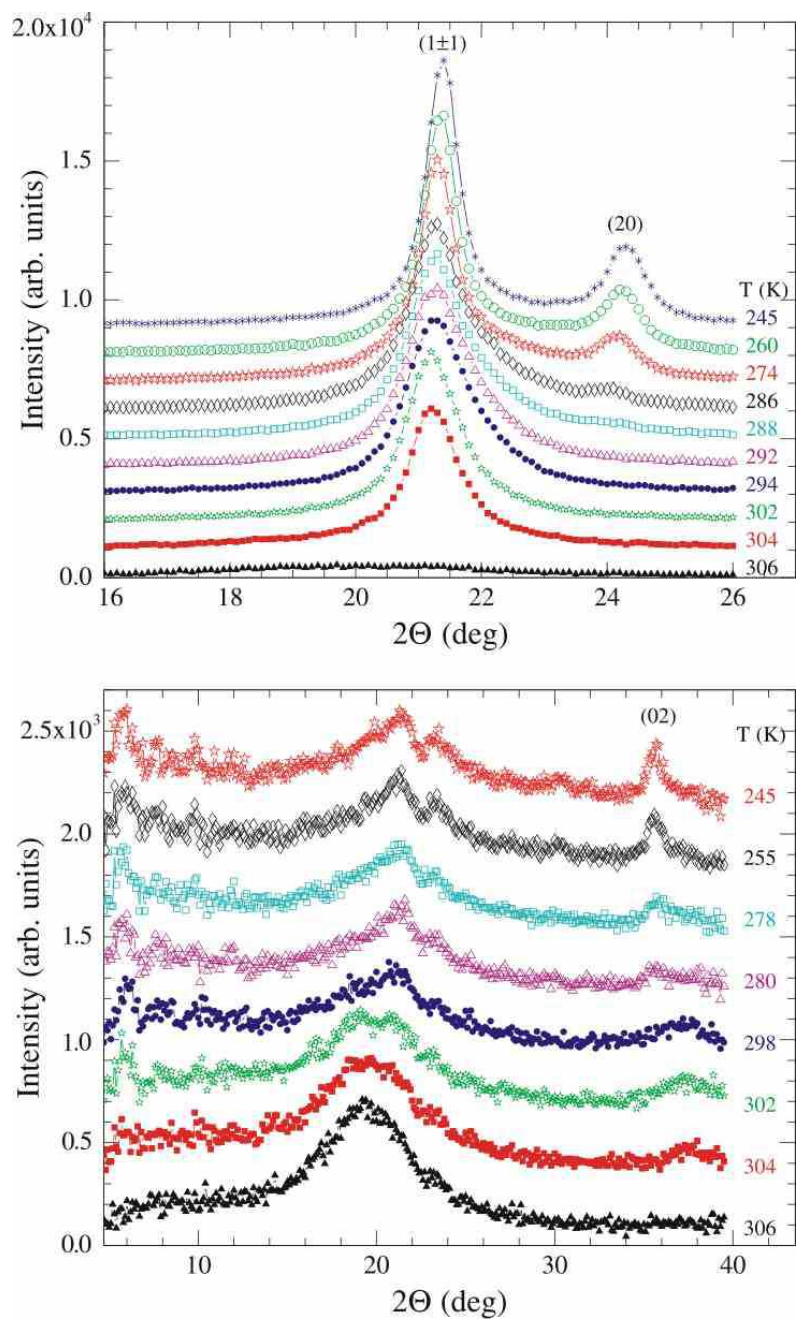

FIG. 5: (Color online) Upper panel: Diffraction pattern of $\mathrm{q}_{\mathrm{p}}$-scans of pore confined $\mathrm{C}_{16} \mathrm{H}_{33} \mathrm{OH}$ while cooling at selected temperatures. Lower panel: Diffraction pattern of $\mathrm{q}_{\mathrm{s}}$-scans at selected temperatures while cooling. The leading in-plane reflections are barely visible. Moreover, at lower angles $(2 \theta \leq$ 10) there are very weak and diffuse layering reflections.

phase, similarly as has been found for n-alkanes 19 and for other orientational disordered, plastic phases under spatial confinement $\underline{\underline{4}}$.

Since the pores were completely filled at higher temperatures, when hexadecanol is in its liquid state, the pore filling at low temperatures does not consist only of bi-layer crystals: the change of volume at the $\mathrm{R}_{I I}$-C phase transition is about $10 \%$ (see Fig. 7), so that there are voids and/or molecules that are not part of a bi-layer crystal. However, our experiments do not give us information about their spatial arrangement. 


\begin{tabular}{|l||c|c|c|c|}
\hline & fluid - $\mathrm{R}_{I I}$ & $\mathrm{R}_{I I}-\mathrm{C}$ & fluid - $\mathrm{R}_{I I}$ & $\mathrm{R}_{I I}-\mathrm{C}$ \\
\hline \hline confined (cooling) & 304 & 291 & & \\
\hline confined (heating) & 312 & 293 & & \\
\hline bulk & & & 322 & 310 \\
\hline
\end{tabular}

TABLE II: Transition temperatures as determined by x-ray experiments of confined and bulk $\mathrm{C}_{16} \mathrm{H}_{33} \mathrm{OH}^{35}$. They agree with those inferred from IR - measurements $\left(\mathrm{CH}_{2}\right.$-scissoring vibration), see Figs. 10 and 11

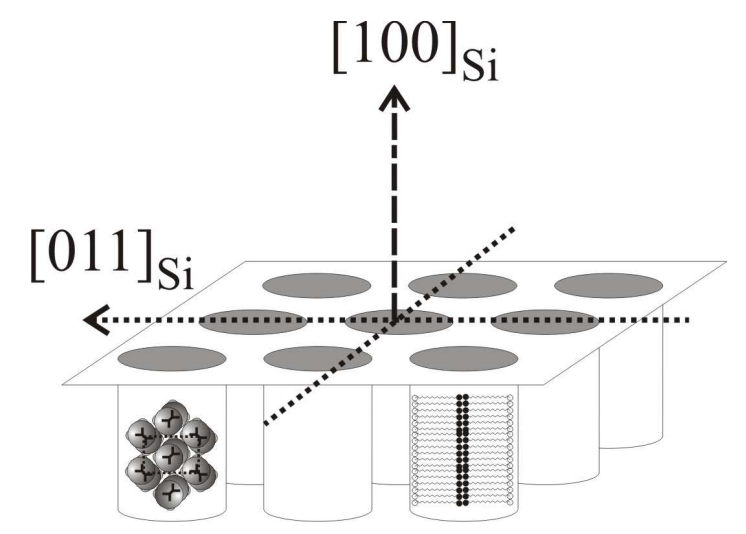

FIG. 6: Sketch of the orientation of pore condensed $\mathrm{C}_{16} \mathrm{H}_{33} \mathrm{OH}$ in the porous sheet. Left pore: view on the rectangular in-plane arrangement of the molecules. Right pore: bi-layered crystal; the long molecule axis are oriented perpendicular to the pore axes. At maximum two bi-layers fit into a pore.

\section{B. Molecular dynamics}

The dynamics of bulk- $\mathrm{C}_{16} \mathrm{H}_{33} \mathrm{OH}$ has already been investigated in IR-measurements in the past $\stackrel{31,33}{ }$. In order to show later on how the molecular dynamics is affected by spatial confinement on the nm-scale, we display some of our bulk spectra in the following.

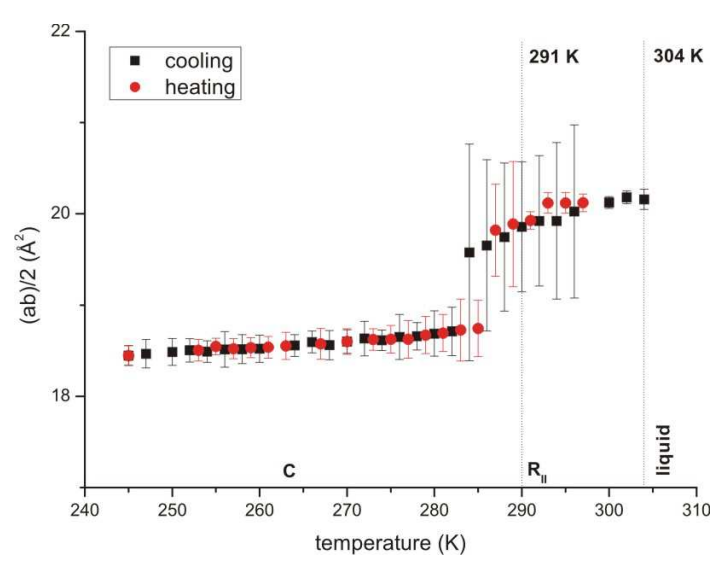

FIG. 7: (color online). Temperature dependence of the area per molecule $A$ for $\mathrm{C}_{16} \mathrm{H}_{33} \mathrm{OH}$ confined in porous silicon.
Here we focus on two characteristic vibrations, the $\mathrm{OH}$-stretching and the $\mathrm{CH}_{2}$-scissoring vibration.

Figs. $[8 \mathrm{a}$ ) and $9 \mathrm{a}$ ) show the bulk spectra of the $\mathrm{OH}-$ stretching-band in the respective phases (compare with Figs. 2(4). In the liquid state (above $322 \mathrm{~K}$ ) the peak maximum is located at about $3345 \mathrm{~cm}^{-1}$. A decrease of temperature below $321 \mathrm{~K}$ yields a shift of the peak position to about $3325 \mathrm{~cm}^{-1}$ indicating the molecular rearrangement in the $R_{I I}$ phase. A further decrease of temperature below $310 \mathrm{~K}$ results in a splitting into two peaks at approximately $3310 \mathrm{~cm}^{-1}$ and $3220 \mathrm{~cm}^{-1}$. Confined $\mathrm{C}_{16} \mathrm{H}_{33} \mathrm{OH}$ shows a different behavior. There is only one peak in the whole temperature range, the position of which changes reflecting the transition between liquid phase and $R_{I I}$ phase as well as between $R_{I I}$ phase and C phase (see Figs. $8 \mathrm{~b}$ and $9 \mathrm{~b}$ ).

The fact that the $\mathrm{OH}$-band of bulk $\mathrm{C}_{16} \mathrm{H}_{33} \mathrm{OH}$ splits at low temperatures while no splitting is observed under confinement confirms the structural differences already observed in the x-ray experiment. For example, Tasumi et. al have studied bulk alcohols $\mathrm{C}_{n} \mathrm{H}_{2 n+1} \mathrm{OH}$ from $n=11-37$ using infrared spectroscopy 33 , Ventòla et al. alcohols with $n=17-20^{34}$. Those alcohols showing at low temperatures ( $\mathrm{C}$ phase) the monoclinic $\gamma$-form, such as $\mathrm{C}_{16} \mathrm{H}_{33} \mathrm{OH}$, exhibit the splitting of the OH-band, while those that take the orthorhombic $\beta$-form show a single peak. This is due to differences in the spatial arrangement of the hydrogen bonds as well as in the distances of neighboring O-atoms: in the crystalline $\gamma$-form, where the molecule axis are tilted (see Fig. 21), the molecules show an all trans conformation, and the intra-layer O-distance $(\simeq 2.74 \AA)$ differs from the interlayer O-distance $(\simeq 2.69 \AA)$. However, in the orthorhombic $\beta$-form (Fig. (3) trans- and gauche-molecules alternate and the intra-layer O-distance $(2.73 \AA)$ nearly equals the inter-layer O-distance $(2.72 \AA)$, so that the splitting is suppressed ${ }^{33}$. Therefore, the observed $\mathrm{OH}-$ band splitting for bulk $\mathrm{C}_{16} \mathrm{H}_{33} \mathrm{OH}$ shows the presence of the $\gamma$-form. Either the whole bulk material exhibits the $\gamma$-form or there is a mixture of $\gamma$ - and $\beta$-crystallites. The latter case is frequently observed ${ }^{33.34}$ : in fact, in the range of wavenumbers from $1150 \mathrm{~cm}^{-1}$ to $950 \mathrm{~cm}^{-1}$, where C-C stretching vibrations are visible, we see indications for a superposition of both forms (not shown). On the other hand, pore confined $\mathrm{C}_{16} \mathrm{H}_{33} \mathrm{OH}$ shows no $\mathrm{OH}$-band-splitting at low temperatures. This reflects that the molecular arrangement doesn't transform in the monoclinic $\gamma$-form but remains in an orthorhombic structure, i. e. only the $\beta$-form is present (compare Figs. 2 and 3). This result is in agreement with the x-ray data presented above. Upon cooling, both the bulk and the confined hexadecanol pass from an hexagonal $R_{I I^{-}}$phase into a crystalline phase. The bulk material undergoes a stronger structural change, $i$. e. there is a mixture of the orthorombic $\beta$ - and the monoclinic $\gamma$-form. The latter one is suppressed under confinement, so that only the $\beta$-form remains, which 
is quite similar to the hexagonal structure of the $\mathbf{R}_{I I}$-phase: the fact that the crystallites have to fit into nanopores of irregular shape might favor the geometrically more simple $\beta$-form ${ }^{3.39}$ (see Fig. 6).
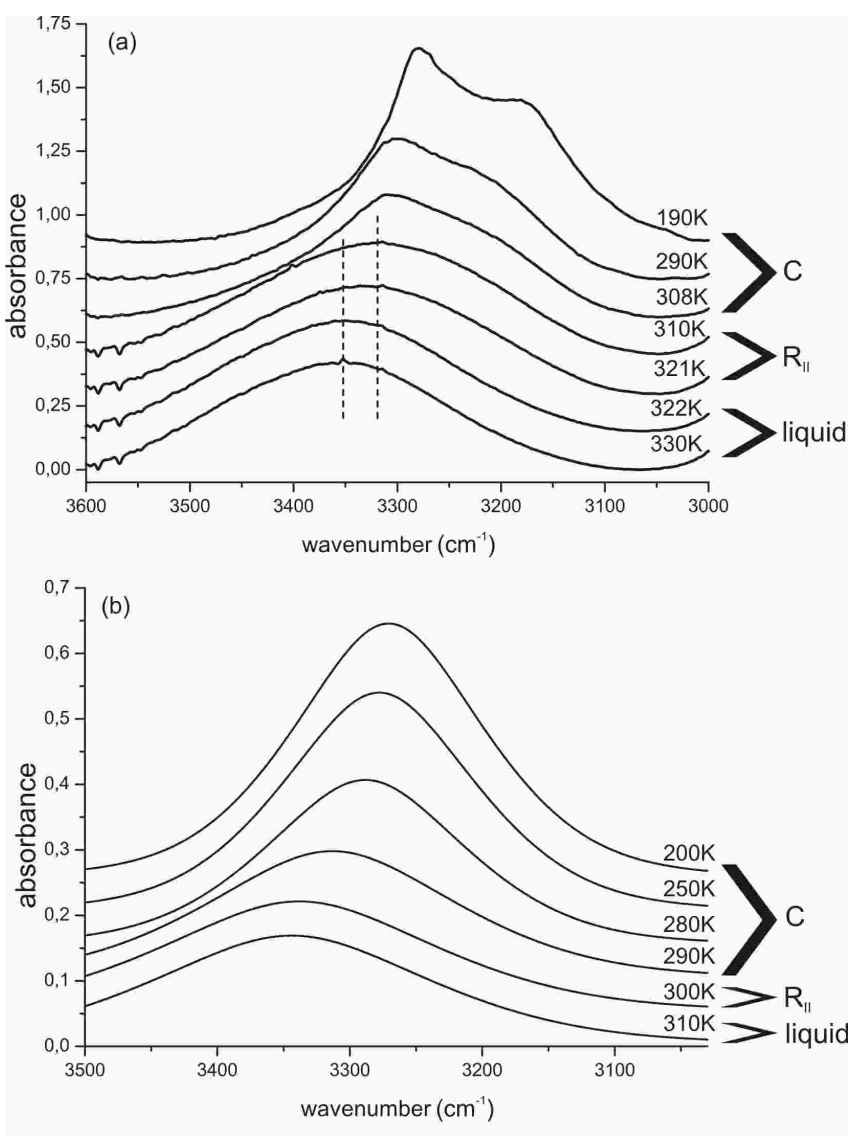

FIG. 8: (a) IR spectrum in the $\mathrm{OH}$ - stretching range for bulk $\mathrm{C}_{16} \mathrm{H}_{33} \mathrm{OH}$. At lower temperatures the peak shifts to lower wavenumbers and then splits into two peaks. (b) Spectrum for confined $\mathrm{C}_{16} \mathrm{H}_{33} \mathrm{OH}$, where no splitting is visible.

Now let us turn towards the scissor-vibration of the $\mathrm{CH}_{2}$ groups (bending mode) that will give us information about inner-molecular and inter-molecular force constants. The spectra are shown in Fig. 10. At first, we want to discuss the bulk material. At high temperatures (liquid state) a superposition of two peaks at $1467 \mathrm{~cm}^{-1}$ and $1460 \mathrm{~cm}^{-1}$ is observed. In the intermediate temperature range $\left(R_{I I}\right.$ phase; see Fig. (4) the intensity of the peak labeld "1" increases strongly. At low temperatures (C phase; see Fig. 2) this band splits up into two peaks. The latter transition can be clearly seen in Figs. 117) and 12 ) , where we display the peak positions and intensities as a function of temperature. The results are similar to those obtained for the bulk state of n-paraffines, that apart from the missing OH-group are similar in their structure, i. e. that have the same $\mathrm{CH}_{2}$-backbone ${ }^{40}$. In IR-spectra only one $\mathrm{CH}_{2}$-scissoring-band is observed at high temperatures, i. e. intra-molecular interactions of the $\mathrm{CH}_{2}$-groups are too small to lead to a series of distinct
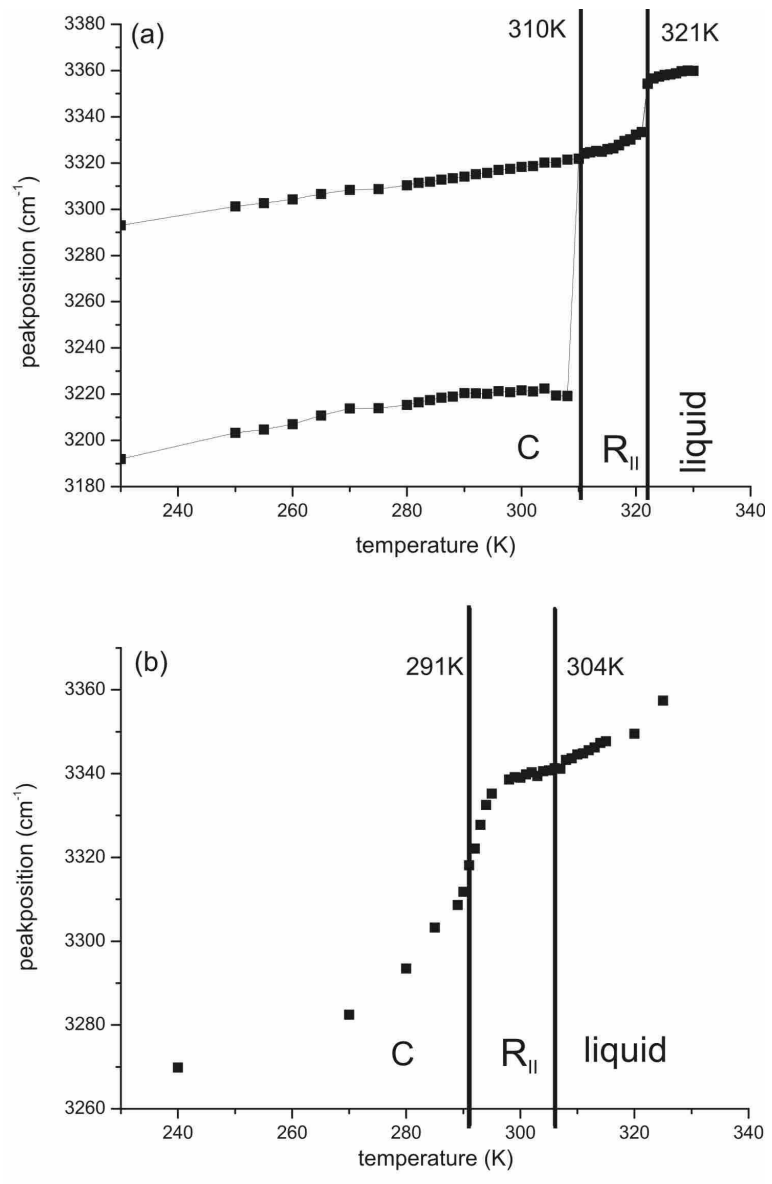

FIG. 9: (a) Wavenumber $\omega / 2 \pi c$ of the $\mathrm{OH}$ - stretching peak vs temperature for bulk $\mathrm{C}_{16} \mathrm{H}_{33} \mathrm{OH}$ [compare with Fig. 83)]. Three different phases are visible: a) above $321 \mathrm{~K}$, b) from 310 to $321 \mathrm{~K}$, where the peak position appears at lower wavenumbers and c) below $310 \mathrm{~K}$ where the peak splits up into two peaks. (b) Wavenumber of the $\mathrm{OH}$ - stretching peak for confined $\mathrm{C}_{16} \mathrm{H}_{33} \mathrm{OH}$ (compare with Fig. 8p). The transition between the $\mathrm{C}$ and the $\mathrm{R}_{I I}$ phase seems to be smeared in a range around $T=291 \pm 5 \mathrm{~K}$. The $\mathrm{R}_{I I}$-liquid transition does not affect the $\mathrm{OH}$-stretching.

peaks. The band splitting at low temperatures has been attributed to inter-molecular interactions (see Ref $\underline{40}$ and text below).

Qualitatively, a behavior similar to that of the bulk state is observed for confined $\mathrm{C}_{16} \mathrm{H}_{33} \mathrm{OH}$ (see Fig. 10 b). In the high-temperature liquid phase two overlapping peaks are visible. The stronger one, i. e. that at higher wavenumbers, undergoes an increase in intensity at about $304 \mathrm{~K}$ (see Fig. 12b), indicating the transition from the liquid phase to the $R_{I I}$ phase, while the secondary peak at lower wavenumbers gets weaker and finally disappears. At the second transition temperature of $T=291 \mathrm{~K}$ the remaining strong peak splits (see also Fig. 11b). The separation is not as distinct as for bulk material. These transition temperatures, $T=304 \mathrm{~K}$ and $T=291 \mathrm{~K}$ (see Figs. 11b and 12b), agree well with 
those obtained via $\mathrm{x}$-ray measurements (compare with Table (II).
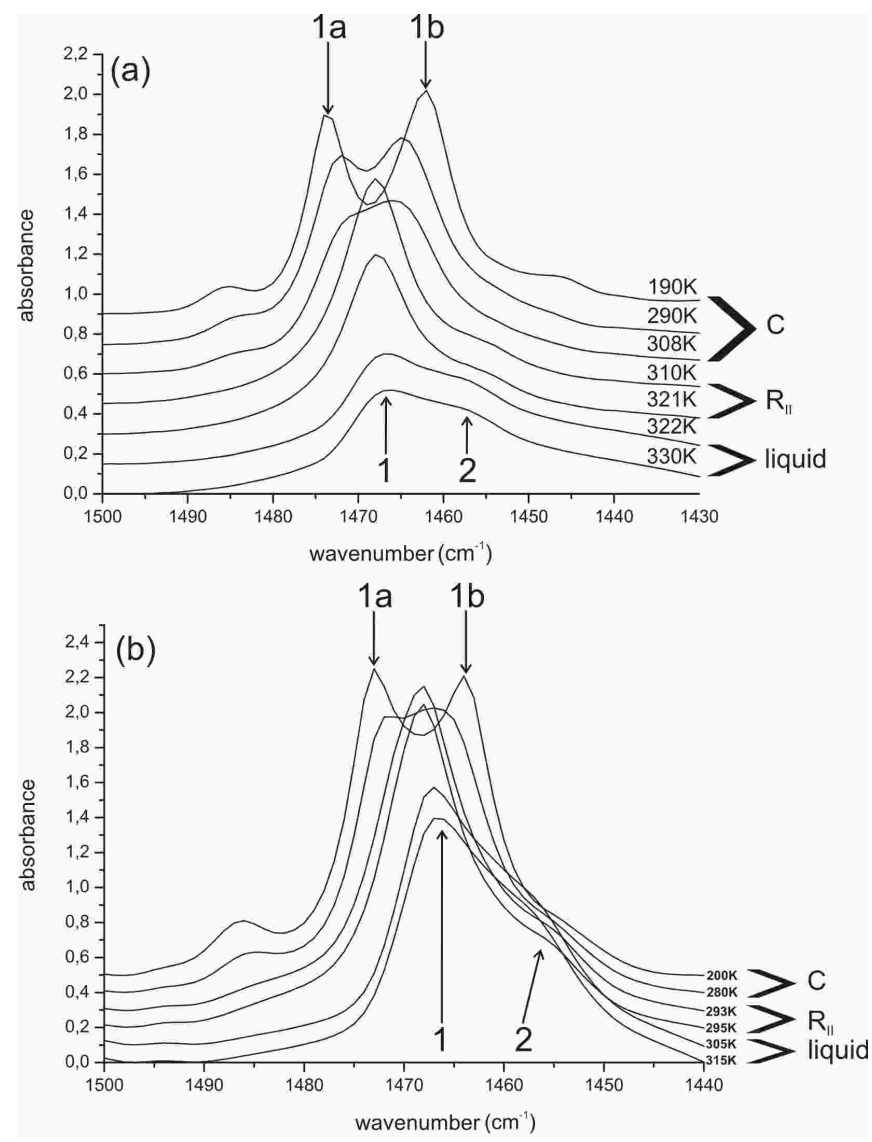

FIG. 10: (a) IR spectrum showing the $\mathrm{CH}_{2}$ scissor-vibration for bulk $\mathrm{C}_{16} \mathrm{H}_{33} \mathrm{OH}$ at various temperatures. (b) IR spectrum showing the $\mathrm{CH}_{2}$ scissor-vibration of $\mathrm{C}_{16} \mathrm{H}_{33} \mathrm{OH}$ confined in mesoporous $\mathrm{Si}$ at various temperatures.

In the following we want to analyze the dynamics of the $\mathrm{CH}_{2}$-groups in order to check whether it is affected by the geometric confinement, e. g. by an interaction with the pore surfaces, by the limited number of neighboring molecules (finite-size-effects) or by structural changes. In a first approximation we can assume that it is not affected by the stretching of the $\mathrm{OH}$ - groups. On the one hand, there is the scissor vibration, where the angle $\alpha$ between the two $\mathrm{CH}$-bonds oscillates around its equilibrium value $\alpha=109.47^{\circ}$ (see Fig. 13). In addition, symmetric and asymmetric stretching vibrations of the $\mathrm{CH}$-bonds are observable (for the values see Table III). Let $f_{\alpha}$ and $f_{d}$ denote the respective force constants. These can be calculated from the measured vibration frequencies using Eqs. (26)-(28) (see Appendix A; the difference in calculating $f_{\alpha}$ via Eq. (27) or Eq. (28) is below $3.5 \%$ confirming that the innermolecular coupling terms can be neglected). Table III shows the results for the liquid and the $R_{I I}$ phase. Neither the phase transition liquid $\rightarrow \mathrm{R}_{I I}$ nor geometrical
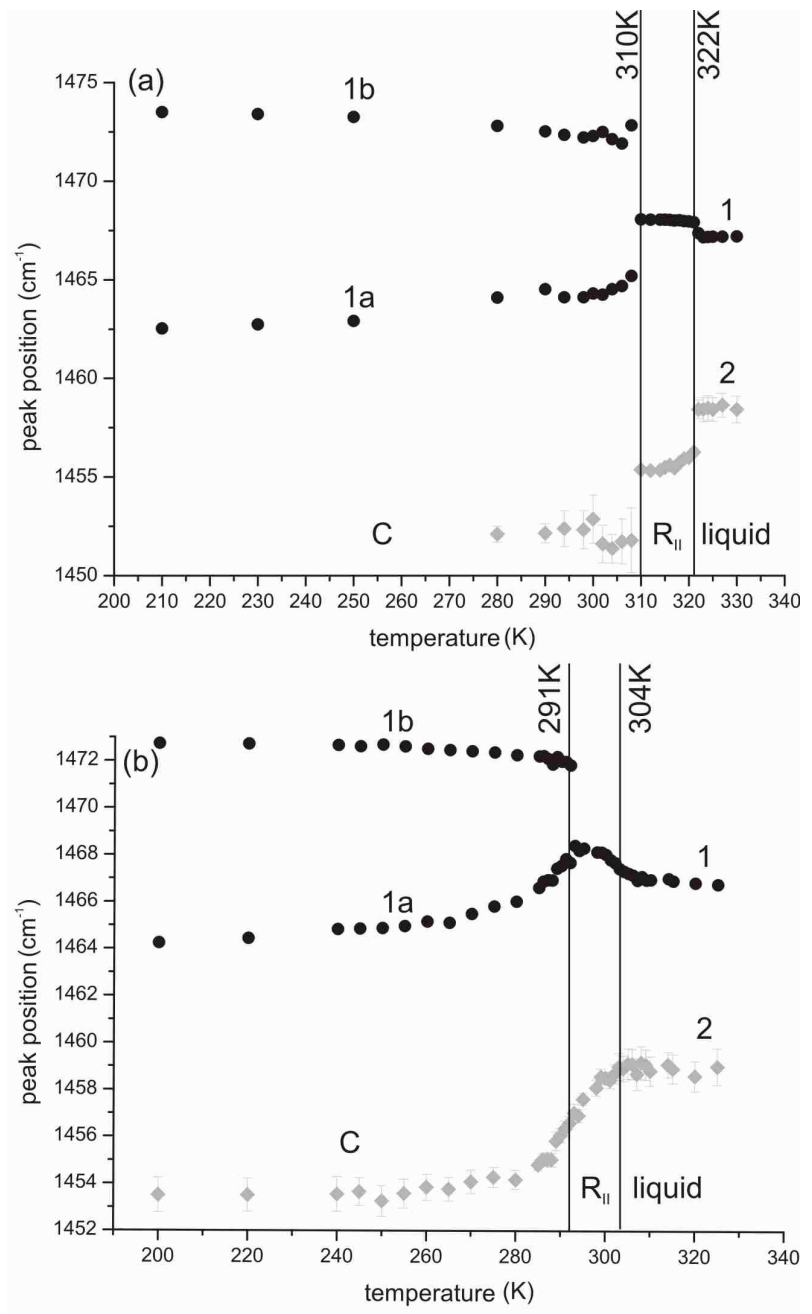

FIG. 11: Wavenumber of the $\mathrm{CH}$ - scissor peak vs temperature for (a) bulk $\mathrm{C}_{16} \mathrm{H}_{33} \mathrm{OH}$ and (b) confined $\mathrm{C}_{16} \mathrm{H}_{33} \mathrm{OH}$ (the peak labels refer to Fig. 10 ).

confinement does markely affect the innermolecular constants.

In the $R_{I I}$ phase the molecules rotate about their long axis, so that the primitive cell consists of only one molecule per layer (see Fig. (4). Therefore, no splitting is observed. But in the $\mathrm{C}$ phase (below $310 \mathrm{~K}$ for bulk and below $291 \mathrm{~K}$ for confined $\mathrm{C}_{16} \mathrm{H}_{33} \mathrm{OH}$ ), where the molecules are arranged in a herringbone structure, there are two molecules per layer in the primitive cell. So the symmetry of the arrangement allows a splitting of the scissoring band and obviously the molecular interactions are sufficiently strong that we are able to observe a double peak (see above, Figs. 10 and 11). The strength of interaction depends on the distances between neighboring $\mathrm{H}$-atoms of adjacent chains and can be analyzed using a formalism developed by Snyder (see Ref. $\underline{40}$ and Appendix B). In Fig. 14 we have sketched the orthorhombic lattice of the crystalline $\mathrm{C}_{16} \mathrm{H}_{33} \mathrm{OH}$ subcell (a view on the a-b-plane perpendicular to the molecules axis). In what follows we restrict ourselves to this $\beta$ - 

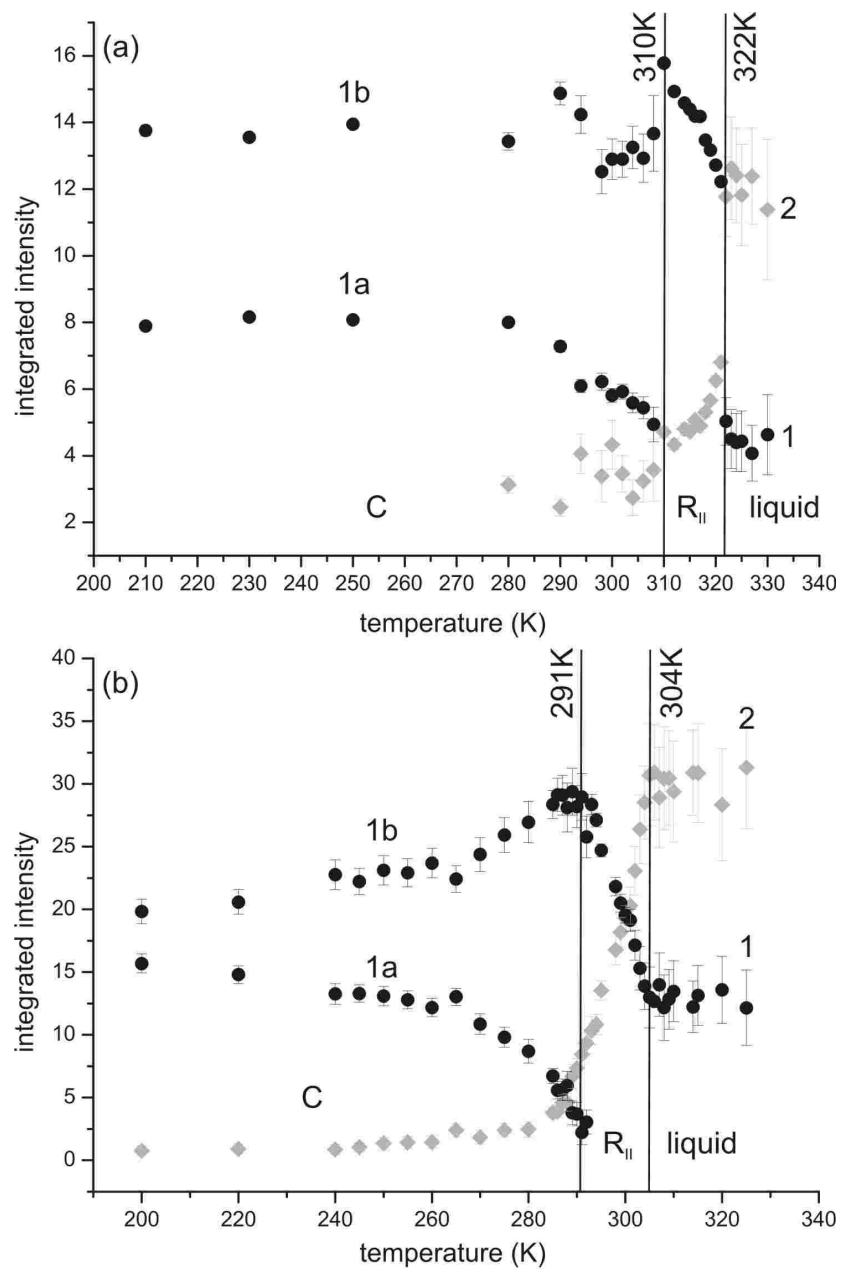

FIG. 12: Integrated intensity of the $\mathrm{CH}$ - scissor peak vs temperature for (a) bulk $\mathrm{C}_{16} \mathrm{H}_{33} \mathrm{OH}$ and (b) confined $\mathrm{C}_{16} \mathrm{H}_{33} \mathrm{OH}$ (the peak labels refer to Fig. 10 ).

form, that is characteristic for confined $\mathrm{C}_{16} \mathrm{H}_{33} \mathrm{OH}$ (a quantitative analysis of bulk $\mathrm{C}_{16} \mathrm{H}_{33} \mathrm{OH}$ is difficult due to the superposition of $\beta$ - and $\gamma$-form). Assuming that the inner force constant $f_{\alpha}$ does not change at the phase transition, the intermolecular force constants $f_{3, j}$ can be evaluated from the observed splitting of the scissor band as described in Appendix B (see Eq. (30)). The values needed are the lattice parameters (see Table I) and the herringbone angle $\zeta$ between the projection of the backbone and the $a$-axis (see Fig. 114). The latter one is determined via Eq. (29) and the measured intensities of the two $\mathrm{CH}_{2}$-scissoring-peaks. For confined $\mathrm{C}_{16} \mathrm{H}_{33} \mathrm{OH}$ we have $I_{a}=13.29$ and $I_{b}=22.23$ yielding an angle of $\zeta=37.7^{\circ}$ (see Fig. $12 \mathrm{~b}$ for $T=245 \mathrm{~K}$ ). We display the intermolecular force constants in Table IV For comparison, we also list literature values for an alkane, $\mathrm{C}_{23} \mathrm{H}_{48}$ at $90 \mathrm{~K}$, which have been evaluated in the same way $\underline{40}$. This alkane and $\mathrm{C}_{16} \mathrm{H}_{33} \mathrm{OH}$ exhibit a similar structure: The backbones of the molecules consist of the same $\mathrm{CH}_{2}$ units and both take the $\beta$-form at low-temperatures. In addition, also the values of the lattice constants for

\begin{tabular}{|c||c|c|c|c|}
\hline \multicolumn{1}{|c||}{} & \multicolumn{2}{c|}{ bulk } & \multicolumn{2}{c|}{ confinement } \\
\cline { 2 - 5 } & liquid & $R_{I I}$ & liquid & $R_{I I}$ \\
\hline \hline scissor $\left[\mathrm{cm}^{-1}\right]$ & 1467 & 1467 & 1467 & 1467 \\
\hline sym. stretch $\left[\mathrm{cm}^{-1}\right]$ & 2854 & 2851 & 2854 & 2851 \\
\hline assym. stretch $\left[\mathrm{cm}^{-1}\right]$ & 2927 & 2921 & 2924 & 2918 \\
\hline$f_{d}[\mathrm{~N} / \mathrm{m}]$ & 455 & 453 & 454 & 452 \\
\hline$f_{\alpha}[\mathrm{N} / \mathrm{m}]$ & $56 \pm 1$ & $56 \pm 1$ & $57 \pm 1$ & $57 \pm 1$ \\
\hline
\end{tabular}

TABLE III: Wavenumbers $\bar{\nu}=\omega /(2 \pi c)$ (with $\omega$ being the angular frequency and $c$ the speed of light) and resulting stretching and bending force constants in the liquid and $R_{I I}$ phase of bulk and confined $\mathrm{C}_{16} \mathrm{H}_{33} \mathrm{OH}$. $f_{\alpha}$ has been evaluated using both the Eq. (27) and the Eq. (28). The difference yields the specified uncertainty. $f_{\alpha}$ are in units of $N / m$ (see Eq. (5) in Appendix A and Ref. $\left.{ }^{41}\right)$. To get $f_{\alpha}$ in units $N \mathrm{~m} / \mathrm{rad}^{2}$ one has to multiply $f_{\alpha}$ with $\mathrm{d}^{2}$, where $d=1,09 \cdot 10^{-10} \mathrm{~m}$ is the $\mathrm{CH}$ bond length.

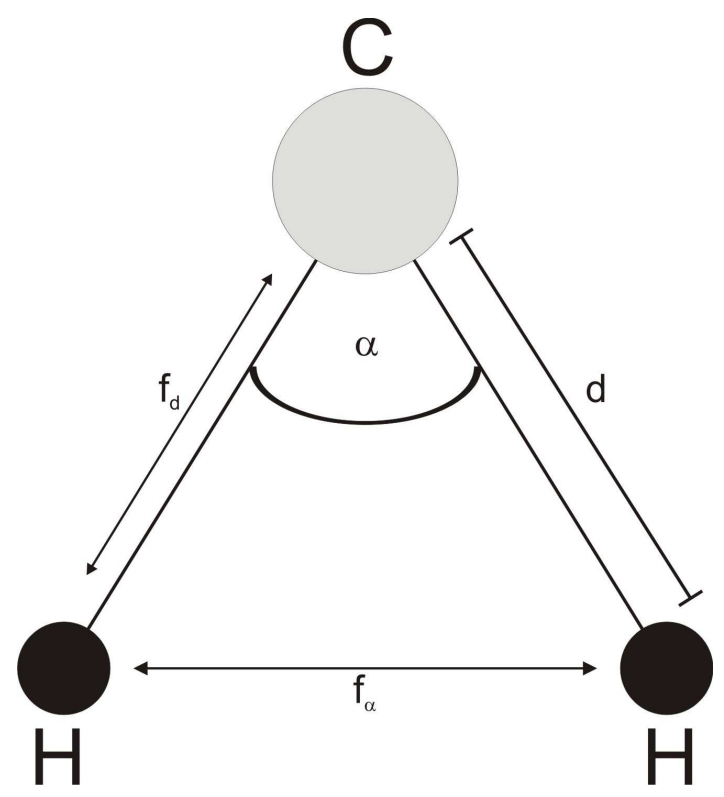

FIG. 13: $\mathrm{CH}_{2}$ molecules with $\mathrm{C}-\mathrm{H}$ bondlength d, H-C-H angle $\alpha$ and the resulting inner force constants $f_{d}$ and $f_{\alpha}$

$\mathrm{C}_{23} \mathrm{H}_{48}, a=7.45 \AA$ and $b=4.96 \AA$, are close to those of $\mathrm{C}_{16} \mathrm{H}_{33} \mathrm{OH}$ (see Tab. \). Due to this structural similarity the intermolecular distances listed in Table [V] are similar, however, the respective force constants differ slightly by 10 to $20 \%$. This is mainly due to the orientation of $\mathrm{CH}_{2}$-groups (the projection of the backbones on the ab-plane) characterized by the herringbone angle $\zeta$. For $\mathrm{C}_{16} \mathrm{H}_{33} \mathrm{OH} \zeta=37.7^{\circ}$ holds, for the alkane $\zeta=42^{\circ}$.

This difference is probably due to the presence of polar $\mathrm{OH}$-groups in $\mathrm{C}_{16} \mathrm{H}_{33} \mathrm{OH}$ that are strongly interacting and thus have an impact on the molecular orientation. The above comparison confirms once again that confined $\mathrm{C}_{16} \mathrm{H}_{33} \mathrm{OH}$ takes the $\beta$-form in contrast to the bulk material ( $\gamma$ - and $\beta$-form).

In order to assess the validity of our analysis, we 


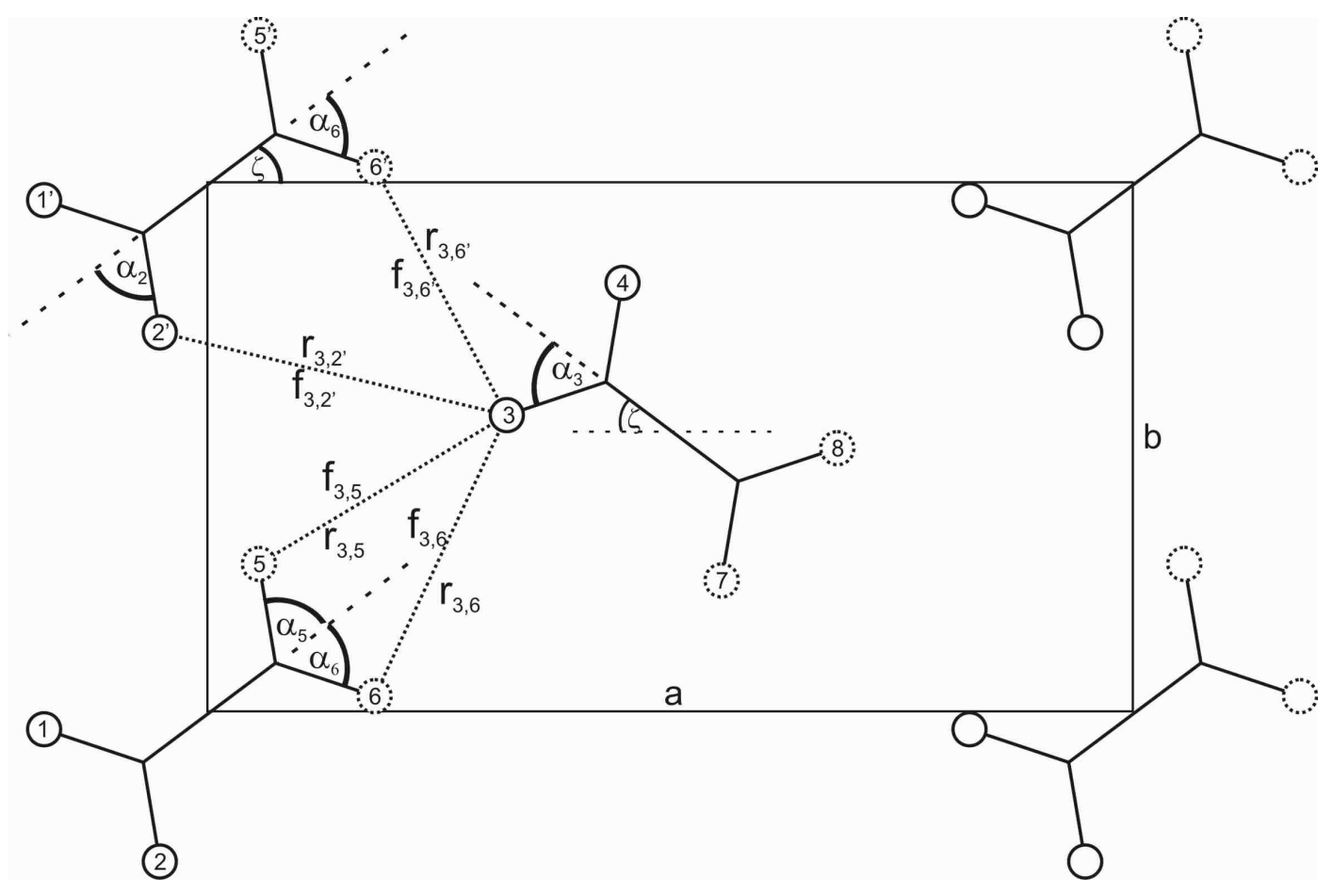

FIG. 14: Lattice of $\mathrm{C}_{16} \mathrm{H}_{33} \mathrm{OH}$ in the $\mathrm{C}$ phase of the $\beta$-form, i. e. a view on the a-b-plane perpendicular to the molecules axis (compare with Fig. 3). Solid circles are H-atoms in the same plane, dashed circles are H-atoms lying in a plane above.

also calculate the theoretical band splitting of the $\mathrm{CH}_{2}-$ scissoring vibration and compare it with the measured values. Using the values from Table IV as well as Eqs. (33) and (34), we get a theoretical value of $\Delta \bar{\nu}_{\text {calc }}=$ $8.1 \mathrm{~cm}^{-1}$ for confined $\mathrm{C}_{16} \mathrm{H}_{33} \mathrm{OH}$ at $T=245 \mathrm{~K}$. The measured band splitting is $\Delta \bar{\nu}_{\text {meas }}=7.8 \mathrm{~cm}^{-1}$. Therefore, the experimental data is in good agreement with the theory.

\begin{tabular}{|c|c|c|c|c|c|}
\hline \multirow[t]{2}{*}{ H-Atoms } & \multirow[t]{2}{*}{$\begin{array}{c}\text { force } \\
\text { constant }\end{array}$} & \multicolumn{2}{|c|}{$\begin{array}{c}\text { confined } \mathrm{C}_{16} \mathrm{H}_{33} \mathrm{OH} \\
(\text { this work) } \\
\zeta=37.7^{\circ}\end{array}$} & \multicolumn{2}{|c|}{$\begin{array}{c}\text { bulk } \mathrm{C}_{23} \mathrm{H}_{48} \\
\left(\operatorname{Ref}{ }^{40}\right) \\
\zeta=42^{\circ}\end{array}$} \\
\hline & & distances $(\AA)$ & $10^{-21} \mathrm{Nm}$ & distances $(\AA)$ & $10^{-21} \mathrm{Nm}$ \\
\hline $3-2$ & $f_{3,2^{\prime}}$ & 2.85 & -4.674 & 2.79 & -5.837 \\
\hline $3-5$ & $f_{3,5}$ & 2.59 & -2.775 & 2.70 & -2.376 \\
\hline $3-6$ & $f_{3,6}$ & 2.96 & -3.508 & 2.94 & -3.920 \\
\hline $3-6$ & $f_{36^{\prime}}$ & 2.96 & -3.508 & 2.94 & -3.920 \\
\hline
\end{tabular}

TABLE IV: Distances between two neighboring hydrogen atoms and the resulting interaction force constants in the $\mathrm{C}$ phase confined $\mathrm{C}_{16} \mathrm{H}_{33} \mathrm{OH}$ ( $\beta$-form). $\zeta$ denotes the herringbone angle between the projection of the backbone and the a-axis (see Fig. 14). For comparison, we also list literature values for an alkane, $\mathrm{C}_{23} \mathrm{H}_{48}$ at $90 \mathrm{~K}$, that also takes the $\beta$ form ${ }^{40}$. The $\mathrm{CH}_{2}$-backbones of both molecules are similar, but $\mathrm{C}_{16} \mathrm{H}_{33} \mathrm{OH}$ exhibits an additional polar $\mathrm{OH}$-group that affects the orientation angle $\zeta$.

\section{SUMMARY}

We have studied the structure and molecular dynamics of n-hexadecanol confined in nanochannels of mesoporous silicon and of bulk n-hexadecanol in their respective phases (in the order of decreasing temperature: liquid, rotator $\mathrm{R}_{I I}$ and $\mathrm{C}$ ). For this purpose we have performed x-ray and infrared-measurements.

The transition-temperatures for confined $\mathrm{C}_{16} \mathrm{H}_{33} \mathrm{OH}$ are lower than for bulk $\mathrm{C}_{16} \mathrm{H}_{33} \mathrm{OH}(\Delta T \simeq 20 \mathrm{~K}$, see Table III). In addition, under confinement the phase transitions are smeared, probably due to a distribution of pore diameters. Geometrical confinement does not affect the innermolecular force constants of the $\mathrm{CH}_{2}$-scissoring vibration (see Table III) but has an impact on the molecular arrangement. The $\mathrm{R}_{I I}$ phase of both bulk and confined hexadecanol is characterized by an orthorhombic subcell, where the chain axis are parallel to the layer normal (see Fig. (4). However, in the low-temperature C phase there is a fundamental structural difference. While bulk $\mathrm{C}_{16} \mathrm{H}_{33} \mathrm{OH}$ exhibits a polycrystalline mixture of $\beta$ and $\gamma$-forms (see Figs. 2 and 3), geometrical confinement favors a phase closely related to the $\beta$-form: only crystallites with an orthorhombic subcell are formed, where the chain axes are parallel to the bi-layer normal. However, the $\gamma$-form having a monoclinic subcell, in which the chain axis are tilted with respect to the layer normal, is suppressed. A reason for this might be the irregular shape of the nanochannels, into which the crystallites have to fit, favoring the formation of the geometrically 
more simple and less bulky form ${ }^{3,39}$ (see Fig. 6). Since only the pure $\beta$-form is present under confinement, we were able to evaluate the inter-molecular force constants of the $\mathrm{CH}_{2}$-scissor vibration. Also the orientation of the $\beta$-crystallites has been determined: the molecules are arranged with their long axis perpendicular to the pore axis.
1 L. D. Gelb, K. E. Gubbins, R. Radhakrishnan and M. Sliwinska-Bartkowiak, Rep. Prog. Phys. 62 (1999) 1573.

2 C. Alba-Simionesco, B. Coasne, G. Dosseh, G. Dudziak, K.E. Gubbins, R. Radhakrishnan and M.G. SliwinskaBartkowiak, J. Phys. Condens. Mat. 18, R15 (2006).

3 H. K. Christenson, J. Phys. Condens. Mat. 13, R95 (2001).

4 K. Knorr, P. Huber and D. Wallacher, Z. Phys. Chem. 222, 257 (2008).

5 J. Koppensteiner, W. Schranz and M. R. Puica, Phys. Rev. B 78,054203 (2008).

${ }^{6}$ P. Scheidler, W. Kob and K. Binder, Europhys. Lett. 52, 277 (2000).

7 F. Kremer, A. Huwe, M. Arndt, P. Behrens and W. Schwieger, J. Phys. Condens. Matter 11 A175 (1999).

8 C. L. Jackson and G. B. McKenna, J. Non-Cryst. Solids 131-133, 221 (1991).

9 G. Barut, P. Pissis, R. Pelster and G. Nimtz, Phys. Rev. Lett. 80, 3543 (1998).

10 R. Pelster, Phys. Rev. B 59, 9214 (1999).

11 D. Daoukaki, G. Barut, R. Pelster, G. Nimtz, A. Kyritsis and P. Pissis, Phys. Rev. B 585336 (1998).

12 P. Pissis, A. Kyritsis, D. Daoukaki, G. Barut, R. Pelster and G. Nimtz, J. Phys. Condens. Matter 10, 6205 (1998).

13 W. Schranz, M. R. Puica, J. Koppensteiner, H. Kabelka and A. V. Kityk, Europhys. Lett. 79, 36003 (2007).

14 B. Frick, M. Koza and R. Zorn, Eur. Phys. J. E 12, 3 (2003).

15 K. Knorr, D. Wallacher, P. Huber, V. Soprunyuk and R. Ackermann, Eur. Phys. J. E 1251 (2003).

16 D. Wallacher, R. Ackermann, P. Huber, M. Enderle and K. Knorr, Phys. Rev. B 64184203 (2001).

17 P. Huber, D. Wallacher, and K. Knorr, J. Low Temp. Phys. 111, 419 (1998); P. Huber and K. Knorr, Phys. Rev. B 60, 12657 (1999); ; P. Huber, D. Wallacher, K. Knorr, Phys. Rev. B 60, 12666 (1999).

18 P. Huber, V. P. Soprunyuk and K. Knorr, Phys. Rev. E 74, 031610 (2006).

19 A. Henschel, T. Hofmann, P. Huber and K. Knorr, Phys. Rev. E 75, 021607 (2007).

20 R. Montenegro and K. Landfester, Langmuir 19, 5996 (2003).

21 B. Xie, G. Liu, S. Jiang, Y. Zhao and D. Wang, J. Phys. Chem. B 112, 13310 (2008).

${ }^{22}$ R. Valiullin and A. Khokhlov, Phys. Rev. E 73, 051605 (2006).

23 G. Crawford and S. Zumer (Editors), Liquid Crystals in Complex Geometries (Taylor and Francis, London, 1996).

24 A. V. Kityk, M. Wolff, K. Knorr, D. Morineau, R. Lefort and P. Huber, Phys. Rev. Lett. 101, 187801 (2008).

${ }^{25}$ P. Huber, D. Wallacher and J. Albers, K.Knorr, Europhys. Lett. 65, 351 (2004).

26 V. Lehmann, U. Gösele, Appl. Phys. Lett. 58, 856 (1991); V. Lehmann, R. Stengl and A. Luigart, Materials Science and Engineering B 69-70, 11 (2000);

27 X. G. Zhang, J. Electrochem. Soc. 151, C69 (2004);
28 A. G. Cullis, L. T. Canham and P. D. J. Calcott, J. Appl. Phys. 82, 909 (1997).

29 S. Gruener and P. Huber, Phys. Rev. Lett. 100, 064502 (2008).

30 P. Huber, S. Gruener, C. Schaefer, K. Knorr and A. V. Kityk, Eur. J. Phys. Special Topics 141101 (2007).

31 V. Métivaud, A. Lefèvre, L. Ventolà, P. Négrier, E. Moreno, T. Calvet, D. Mondieig and M.A. Cuevas-Diarte, Chem. Mater. 17, 3302 (2005).

32 S. Abrahamsson, G. Larsson and E. von Sydow, Acta Cryst. 13, 770 (1960).

33 M. Tasumi, T. Shimanouchi, A. Watanabe and R. Goto, Spectrochim. Acta 20, 629 (1964).

34 L. Ventolà, M. Ramirez, T. Calvet, X.Solans, M.A. CuevasDiarte, P. Negrier, D. Mondieig, J.C. van Miltenburg, H.A.J. Oonk, Chem. Mater. 14, 508 (2002).

35 E. B. Sirota and X. Z. Wu, J. Chem. Phys. 105, 7763 (1996).

36 A. Henschel, P. Huber and K. Knorr, Phys. Rev. E 77, 042602 (2008).

37 M. Steinhart, P. Göring, H. Dernaika, M. Prabhukaran, U. Gösele, E. Hempel and T. Thurn-Albrecht, Phys. Rev. Lett. 97, 027801 (2006).

38 P. A. Palibin and A. I. Froiman, Z. Kristallogr. 85, 322 (1933); P. W. Bridgman, Proc. Amer. Acad. Arts Sci. 60 305 (1925).

39 K. Morishige and K. Kawano, J. Chem. Phys. 112, 11023 (2000).

40 R. G. Snyder, J. Mol. Spectroscopy 7, 116 (1961).

41 A. G. Meister and F. F. Cleveland, Am. J. Physics 14, 13 (1946).

42 E. B. Wilson Jr., J. Chem. Physics 7, 1047 (1939).

43 H. Siebert, Anwendungen der Schwingungsspektroskopie in der anorganischen Chemie, Springer-Verlag, Berlin (1966).

44 M. Tasumi, T. Shimanouchi, J. Chem. Phys. 43, 1245 (1965).

45 R. S. Stein, J. Chem. Phys. 23, 734 (1955).

46 D. A. Dows, J. Chem. Phys. 32, 1342 (1960).

47 J. deBoer, Physica 9, 363 (1942). 


\section{Appendix A}

In this section we show how the innermolecular force constants of the $\mathrm{CH}_{2}$-groups can be evaluated using three characteristic vibration frequencies, that are easily measured: the scissor vibration as well as the symmetric and asymmetric $\mathrm{CH}$-bond stretching. For this purpose we apply the Wilson FG - matrix method ${ }^{42}$. We use the notation of Meister and Cleveland for the similar $\mathrm{H}_{2} \mathrm{O}$ molecule ${ }^{41}$ and perform the calculations in the same way.

Fig. 13 shows a single $\mathrm{CH}_{2}$ - molecule. In the following we will neglect the influence of the neighboring molecules on this one. $d=1.09 \AA$ is the length of the $\mathrm{C}-\mathrm{H}$ bond and $\alpha=109.47^{\circ}$ the angle between the two $\mathrm{C}-\mathrm{H}$ bonds 32 . This kind of molecule belongs to the $C_{2_{\nu}}$ point group. This means, there are two vibrations of type $A_{1}$ (symmetric stretching and bending vibration) and one vibration of type $B_{2}$ (asymmetric stretching vibration). The internal coordinates of this molecule are $\Delta d_{1}, \Delta d_{2}$ and $\Delta \alpha . \Delta d_{1}$ and $\Delta d_{2}$ mean changes in the bond length of the two $\mathrm{C}-\mathrm{H}$ - bonds and $\Delta \alpha$ changes in the angle between the two bonds. Therefore we get three symmetry coordinates, two for $A_{1}$ and one for $B_{2}$. If we assume d being the equilibrium $\mathrm{C}-\mathrm{H}$ bond length, then we obtain for the three symmetry coordinates:

$$
\begin{gathered}
R_{1}=\sqrt{\frac{1}{2}} \Delta d_{1}+\sqrt{\frac{1}{2}} \Delta d_{2} \\
R_{2}=\Delta \alpha \cdot d \\
R_{3}=\sqrt{\frac{1}{2}} \Delta d_{1}-\sqrt{\frac{1}{2}} \Delta d_{2}
\end{gathered}
$$

Now, we have to calculate the $\mathbf{F}$ matrix, related to the potential energy, and the $\mathbf{G}$ matrix related to the kinetic energy. The potential energy can be written as

$$
2 V=\sum f_{i k} r_{i} r_{k}
$$

and with the internal coordinates

$$
\begin{aligned}
2 V= & f_{d}\left[\left(\Delta d_{1}\right)^{2}+\left(\Delta d_{2}\right)^{2}\right] \\
& +f_{\alpha}(d \Delta \alpha)^{2}+2 f_{d \alpha}\left(\Delta d_{1}+\Delta d_{2}\right)(d \Delta \alpha) \\
& +2 f_{d d}\left(\Delta d_{1}\right)\left(\Delta d_{2}\right)
\end{aligned}
$$

Now we set $d_{1}=d_{2}=d$ and write Eq. (5) as

$$
2 V=\sum F_{j l} R_{j} R_{l}
$$

with $F_{j l}=F_{l j}$. In matrix form, Eqs. (4) and (5) become

$$
2 V=\mathbf{r}^{\prime} \mathbf{f r}
$$

and

$$
2 V=\mathbf{R}^{\prime} \mathbf{F R}
$$

$\mathbf{r}^{\prime}$ and $\mathbf{R}^{\prime}$ are the transposes of $\mathbf{r}$ and $\mathbf{R}$. With Eqs. (7) + (8)

$$
\mathbf{r}^{\prime} \mathbf{f r}=\mathbf{R}^{\prime} \mathbf{F R}
$$

The $R_{i}$ 's are linear combinations of the $r_{i}$ 's

$$
\begin{aligned}
R_{i} & =\sum_{k} U_{i k} r_{k} \\
\mathbf{R} & =\mathbf{U r}
\end{aligned}
$$

Since the $R_{i}$ 's are orthogonal and normalized, then $\mathbf{U}^{-1}=\mathbf{U}^{\prime}$ and

$$
\begin{aligned}
\mathbf{r} & =\mathbf{U}^{\prime} \mathbf{R} \\
\mathbf{r}^{\prime}=\left(\mathbf{U}^{\prime} \mathbf{R}\right)^{\prime} & =\mathbf{R}^{\prime} \mathbf{U}
\end{aligned}
$$

This means with Eqn. (10)

$$
\begin{array}{r}
\mathbf{R}^{\prime}\left(\mathbf{U f U}^{\prime}\right) \mathbf{R}=\mathbf{R}^{\prime} \mathbf{F R} \\
\mathbf{F}=\mathbf{U f U}^{\prime}
\end{array}
$$

The $\mathbf{F}$ matrix is

\begin{tabular}{c|ccc} 
& $\Delta d_{1}$ & $\Delta d_{2}$ & $\Delta \alpha$ \\
\hline$\Delta d_{1}$ & $f_{d}$ & $f_{d d}$ & $d f_{d \alpha}$ \\
$\Delta d_{2}$ & $f_{d d}$ & $f_{d}$ & $d f_{d \alpha}$ \\
$\Delta \alpha$ & $d f_{d \alpha}$ & $d f_{d \alpha}$ & $d^{2} f_{\alpha}$
\end{tabular}

The $\mathbf{U}$ matrix for type $A_{1}$ is

\begin{tabular}{c|ccc}
$A_{1}$ & $\Delta d_{1}$ & $\Delta d_{2}$ & $\Delta \alpha$ \\
\hline$R_{1}$ & $\sqrt{\frac{1}{2}}$ & $\sqrt{\frac{1}{2}}$ & 0 \\
$R_{2}$ & 0 & 0 & 1
\end{tabular}

and for $B_{2}$

$$
\begin{array}{c|ccc}
B_{2} & \Delta d_{1} & \Delta d_{2} & \Delta \alpha \\
\hline R_{3} & \sqrt{\frac{1}{2}} & -\sqrt{\frac{1}{2}} & 0
\end{array}
$$

So, for the type $A_{1}$ the $\mathbf{F}$ matrix is

$$
\mathbf{F}_{A_{1}}=\mathbf{U f U}^{\prime}=\left(\begin{array}{cc}
F_{11} & F_{12} \\
F_{21} & F_{22}
\end{array}\right)=\left(\begin{array}{cc}
f_{d}+f_{d d} & \sqrt{2} d f_{\alpha} \\
\sqrt{2} d f_{\alpha} & d^{2} f_{\alpha}
\end{array}\right)
$$

and for the $B_{2}$ type

$$
\mathbf{F}_{B_{2}}=\left(F_{33}\right)=\left(f_{d}-f_{d d}\right)
$$

The exact derivation of the $\mathbf{G}$ matrix shouldn't be shown here. It can be gleaned by Meister and Cleveland ${ }^{41}$. Only the most important steps shall be explained here.

If only non-degenerate vibrations are present, the elements of the kinetic energy matrix can be written as

$$
G_{j l}=\sum_{p} \mu_{p} g_{p} \mathbf{S}_{j}^{(t)} \mathbf{S}_{l}^{(t)}
$$


where $\mathrm{j}$ and $\mathrm{l}$ refer to symmetry coordinates used in determining the $\mathbf{S}$ vector, $p$ refer to a set of equivalent atoms, a typical one of the set being t. $\mu_{p}$ is the reciprocal of the mass of the typical atom $t_{p}$ and $g_{p}$ is the number of equivalent atoms in the $p$ th set. The $\mathbf{S}$ vector is given by

$$
\mathbf{S}_{j}^{(t)}=\sum_{k} U_{j k} s_{k t}
$$

where $j, U_{j} k$ and $\sum_{k}$ have the same meaning as above. $s_{k t}$ can be expressed in terms of unit vectors along the chemical bonds and depends on the changes in the bond length or the angle between the bonds. So, the $\mathbf{G}$ matrix for the $A_{1}$ vibration type has the form

$$
\begin{aligned}
\mathbf{G}_{A_{1}} & =\left(\begin{array}{ll}
G_{11} & G_{12} \\
G_{21} & G_{22}
\end{array}\right) \\
& =\left(\begin{array}{cc}
\mu_{H}+\mu_{C}(1+\cos \alpha) & -\frac{\mu_{C} \sqrt{(2) \sin \alpha}}{d} \\
-\frac{\mu_{C} \sqrt{(2) \sin \alpha}}{d} & \frac{2 \mu_{H}+\mu_{C}(1-\cos \alpha)}{d}
\end{array}\right)
\end{aligned}
$$

and for the $B_{2}$ vibration type

$$
\mathbf{G}_{B_{2}}\left(G_{33}\right)=\left(\mu_{H}+\mu_{C}(1-\cos \alpha)\right)
$$

To determine the frequencies, one has to solve the equation

$$
|\mathbf{G F}-\lambda \mathbf{E}|=0
$$

where $\lambda=\omega^{2}=(\bar{\nu} 2 \pi c)^{2}$ denotes the square of the angular frequency. For the $A_{1}$ type one gets the equation

$$
\begin{gathered}
\lambda^{2}-\lambda\left(F_{11} G_{11}+2 F_{12} G_{12}+F_{22} G_{22}\right) \\
+\left|\begin{array}{ll}
F_{11} & F_{12} \\
F_{21} & F_{22}
\end{array}\right| \cdot\left|\begin{array}{ll}
G_{11} & G_{12} \\
G_{21} & G_{22}
\end{array}\right|=0
\end{gathered}
$$

and for the $B_{2}$ type

$$
\lambda_{3}-F_{33} G_{33}=0
$$

Eq. (22) can be separated with the Vieta expression ${ }^{43}$. Inserting the terms for the $F_{i j}$ and $G_{i j}$, we obtain

$$
\begin{aligned}
\lambda_{1}+\lambda_{2} & =\left(f_{d}+f_{d d}\right)\left[\mu_{C}(1+\cos \alpha)+\mu_{H}\right] \\
& +2 f_{\alpha}\left[\mu_{C}(1-\cos \alpha)+\mu_{H}\right]-4 f_{d \alpha} \mu_{C} \sin \alpha(24) \\
\lambda_{1} \cdot \lambda_{2} & =\left[\left(f_{d}+f_{d d}\right) f_{\alpha}-2 f_{d \alpha}^{2}\right] 2 \mu_{H}\left(2 \mu_{C}+\mu_{H}\right)
\end{aligned}
$$

For Eq. (23) one obtains

$$
\lambda_{3}=\left(f_{d}-f_{d d}\right)\left[\mu_{C}(1-\cos \alpha)+\mu_{H}\right]
$$

Neglecting the coupling constants $f_{d d}$ and $f_{d \alpha}$ allows to evaluate the innermolecular force constants using the measured wave numbers, $\bar{\nu}_{d, s y m}=\sqrt{\lambda_{1}} /(2 \pi c), \bar{\nu}_{\alpha}=$ $\sqrt{\lambda_{2}} /(2 \pi c)$ and $\bar{\nu}_{d, a s y m}=\sqrt{\lambda_{3}} /(2 \pi c)$. Then Eq. (25) yields

$$
f_{d}=(2 \pi c)^{2} \cdot \frac{\bar{\nu}_{d, a s y m}^{2}}{\mu_{C}(1-\cos \alpha)+\mu_{H}}
$$

Inserting this result into Eq. (24) yields

$$
f_{\alpha}=(2 \pi c)^{2} \cdot \frac{\bar{\nu}_{d, s y m}^{2} \cdot \bar{\nu}_{\alpha}^{2}}{\bar{\nu}_{d, a s y m}^{2}} \cdot \frac{\mu_{C}(1-\cos \alpha)+\mu_{H}}{2 \mu_{H}\left(2 \mu_{C}+\mu_{H}\right)}
$$

There is a second possibility to evaluate $f_{\alpha}$, i. e. by inserting Eq. (26) into Eq. (24). This yields

$$
f_{\alpha}=(2 \pi c)^{2} \cdot \frac{\bar{\nu}_{d, s y m}^{2}+\bar{\nu}_{\alpha}^{2}-\bar{\nu}_{d, a s y m}^{2} \cdot \frac{\mu_{C}(1+\cos \alpha)+\mu_{H}}{\mu_{C}(1-\cos \alpha)+\mu_{H}}}{2\left(\mu_{C}(1-\cos \alpha)+\mu_{H}\right)}
$$

Taking the measured wavenumbers listed in Table III and the average angle between the CH-bonds, $\alpha=$ $109.4^{\circ}$, as well as the masses of the atoms, $1 / \mu_{C}=12 u$ and $1 / \mu_{H}=1 u\left(u=1.6606 \cdot 10^{-27} \mathrm{~kg}\right)$, Eqs. (26)-(28) yield the force constants listed in Table III. The difference in calculating $f_{\alpha}$ via Eq. (27) or Eq. (28) is below $3.1 \%$ confirming that the inner-molecular coupling terms can be neglected.

\section{Appendix B}

What follows is a summary of Snyder's derivation of the intermolecular force constants between the $\mathrm{CH}_{2}$ groups of neighboring molecules that gives rise to a splitting of the scissor band at low temperatures ${ }^{40}$. We show how this formalism can be applied to $\mathrm{C}_{16} \mathrm{H}_{33} \mathrm{OH}$. An alternative description can be found in Ref. 44 .

In Fig. 14 we display a hexagonal subcell of $\mathrm{C}_{16} \mathrm{H}_{33} \mathrm{OH}$. While Stein 45 has taken only one pair of neighboring $\mathrm{CH}_{2}$ into account to calculate the splitting of rocking and scissoring bands, Snyder has shown that more pairs have to be included. When we consider the distances of H-atoms from the H-atom no. 3 (see Fig. 14), then all atoms except no. $2^{\prime}, 6^{\prime}, 5$ and 6 have distances larger then $3.7 \AA$. The internal coordinates $\alpha_{i}$ are always half of the angle between the $\mathrm{C}$ - $\mathrm{H}$ bonds of a $\mathrm{CH}_{2}$ molecule. Solid circles are $\mathrm{H}$-atoms in the same plane, dashed circles $\mathrm{H}$-atoms in a plane above or below.

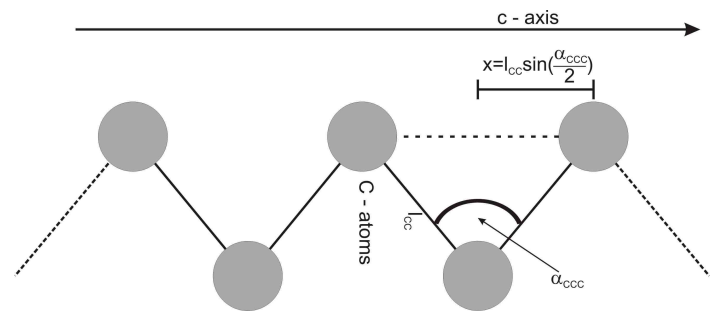

FIG. 15: Lateral view at the long axis of the $\mathrm{C}_{16} \mathrm{H}_{33} \mathrm{OH}$ chain, $\mathrm{x}$ is the projection of the $\mathrm{C}-\mathrm{C}$ distance on the c-axis of the crystal lattice

Now, we want to write the positions of these five H-atoms as a vector. Fig. 15] shows the lateral view of a part of the $\mathrm{C}_{16} \mathrm{H}_{33} \mathrm{OH}$ chain. With values from Abrahamsson ${ }^{32}$ for $l_{C C}=1.545 \AA$ and $\alpha_{C C C}=110.4^{\circ}$, we can calculate 
the distance of the a-b-plane to the corresponding plane above or below with

$$
x=l_{C C} \sin \left(\frac{\alpha_{C C C}}{2}\right)=1.2687 \AA
$$

Assuming that the hydrogen in the central plane has the $c$ component 0 , the hydrogen in the plane above has the component $c=1.2687 \AA$.

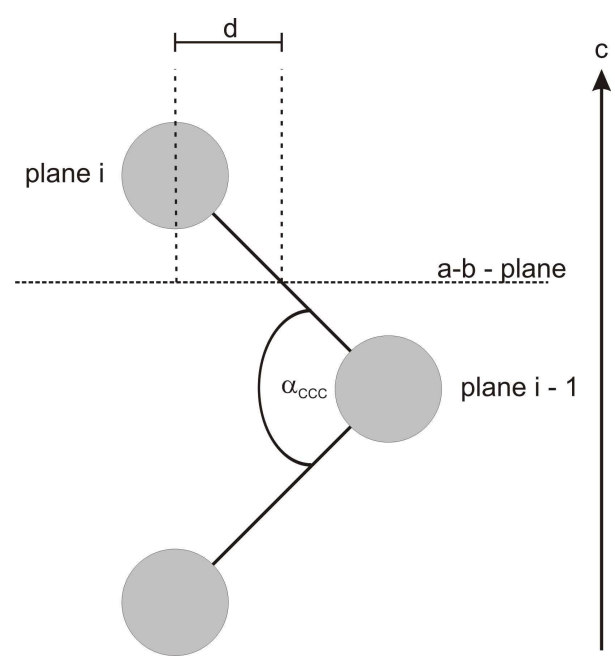

FIG. 16: Lateral view at the long axis of the $\mathrm{C}_{16} \mathrm{H}_{33} \mathrm{OH}$ chain, $\mathrm{d}$ is the projection of half of the $\mathrm{C}-\mathrm{C}$ distance on the a-bplane of the subcell.

The projection of the $\mathrm{C}-\mathrm{C}$ bond in the a-b-plane is according to Fig. 16

$$
d=\frac{l_{C C}}{2} \cos \left(\frac{\alpha_{C C C}}{2}\right)=0.4409 \AA
$$

Taking the point $\mathbf{0}$ for the lower left edge of the ab - plane the five atoms have the coordinates:

$$
\begin{aligned}
& H_{3}=\left(\begin{array}{c}
\frac{a}{2}-d \cos (\zeta)-l \cos \left(\alpha_{3}-\zeta\right) \\
\frac{b}{2}+d \sin (\zeta)-l \sin \left(\alpha_{3}-\zeta\right) \\
0
\end{array}\right) \\
& H_{2^{\prime}}=\left(\begin{array}{c}
-d \cos (\zeta)+l \sin \left(\alpha_{2^{\prime}}-\frac{\pi}{2}+\zeta\right) \\
b-d \sin (\zeta)-l \cos \left(\alpha_{2^{\prime}}-\frac{\pi}{2}+\zeta\right) \\
0
\end{array}\right) \\
& H_{5}=\left(\begin{array}{c}
d \cos (\zeta)-l \sin \left(\alpha_{5}-\frac{\pi}{2}+\zeta\right) \\
d \sin (\zeta)+l \cos \left(\alpha_{5}-\frac{\pi}{2}+\zeta\right) \\
1.2674
\end{array}\right) \\
& H_{6}=\left(\begin{array}{c}
d \cos (\zeta)+l \cos \left(\alpha_{6}-\zeta\right) \\
d \sin (\zeta)-l \sin \left(\alpha_{6}-\zeta\right) \\
1.2674
\end{array}\right) \\
& H_{6^{\prime}}=\left(\begin{array}{c}
d \cos (\zeta)+l \cos \left(\alpha_{6^{\prime}}-\zeta\right) \\
b+d \sin (\zeta)-l \cos \left(\alpha_{6^{\prime}}-\zeta\right) \\
1.2674
\end{array}\right)
\end{aligned}
$$

with $a$ and $b$ being the lattice constants of the crystalline phase. We get the distances between the atom 3 and the other ones (see Fig. 14) with

$$
r_{3 j}=\left|H_{3}-H_{j}\right|
$$

where $j=2^{\prime}, 6^{\prime}, 5,6$ is. The herringbone angle $\zeta$ (see the upper left corner of Fig. 14) can be determined with the relation $\underline{40}$

$$
\frac{I_{a}}{I_{b}}=\tan ^{2} \zeta
$$

where $I_{a}$ is the integrated intensity of the scissoring mode, which is polarized in the a direction (higher mode at $1473 \mathrm{~cm}^{-1}$ ) and $I_{b}$ the one of the mode, which is polarized in the $b$ direction (lower mode at $1462 \mathrm{~cm}^{-1}$ ).

With the distances of two hydrogen atoms $H_{3}$ and $H_{j}$ $\left(=r_{3, j}\right)$ [in our case 3 denotes the central H-atom (see Fig. (14) and $j=2^{\prime}, 5,6,6^{\prime}$ the neighboring $\mathrm{H}$-atoms that interact] we obtain the intermolecular force constants $f_{3, j}$ :

$$
\begin{aligned}
f_{3, j} & =\frac{\partial^{2} V_{H H}}{\partial \alpha_{3} \partial \alpha_{j}} \\
& =\left(\frac{\partial^{2} V_{H H}}{\partial r^{2}}\right)_{r_{3 j}}\left(\frac{\partial r}{\partial \alpha_{3}}\right)\left(\frac{\partial r}{\partial \alpha_{j}}\right)
\end{aligned}
$$

where $V_{H H}$ is the hydrogen repulsion potential introduced by Dows $\underline{46}$ :

$$
V_{H H}=1.2 \cdot 10^{-10} e^{-3.52 r}
$$

with $\mathrm{r}$ in $\AA$.

The values of $\left(\partial^{2} V_{H H} / \partial r^{2}\right)_{r_{i j}}$ are obtained from Eq. (32).

$$
\beta=\frac{\partial^{2} V_{H H}}{\partial r^{2}}=1.486848 \cdot 10^{-9} e^{-3.52 r}
$$

with $\beta$ in $\frac{\mathrm{ergs}}{\AA^{2}}=10^{16} \frac{d y n e}{\mathrm{~cm}}=10^{13} \frac{\mathrm{N}}{\mathrm{m}}$.

The measured intensity ratio (Eq. (29)) allows us to calculate the distances $r_{i j}$ as well as the partial derivatives $\partial r / \partial \alpha_{i}$ (see Eq. (28) and above). Finally, by knowing the intermolecular force constants $f_{3, j}$ from Eq. (32) we can evaluate the band splitting of the scissoring vibration $\stackrel{40}{ }$. For the angular frequencies

$\bar{\nu}_{1}^{2}-\bar{\nu}_{2}^{2}=\left(\frac{1}{2 \pi c}\right)^{2} \cdot \underbrace{G_{a}^{B} \cdot\left\{2 f_{3,2^{\prime}}-2\left(f_{3,6}+f_{3,6^{\prime}}\right)-4 f_{3,5}\right\}}_{\Delta \lambda^{B}}$

holds ${ }^{3}$ with

\footnotetext{
${ }^{3}$ In Snyder's general theory the force constants for the scissoring vibration are denominated as $f_{a}^{3}\left(=f_{3,2^{\prime}}\right), f_{b}^{2}\left(=f_{3,6}+f_{3,6^{\prime}}\right)$ and $f_{b}^{3}\left(=f_{3,5}\right)$.
} 


$$
G_{a}^{B}=\frac{4}{3} Q_{R}^{2} \mu_{C}+Q_{r}^{2} \mu_{H}
$$

Here $1 / \mu_{C}=12 u$ and $1 / \mu_{H}=1 u$ denote the masses of the atoms $\left(u=1.6606 \cdot 10^{-27} \mathrm{~kg}\right), 1 / Q_{R}=1.545 \cdot 10^{-10} \mathrm{~m}$ the $\mathrm{C}-\mathrm{C}$ distance and $1 / Q_{r}=1.09 \cdot 10^{-10} \mathrm{~m}$ the $\mathrm{C}-\mathrm{H}$ distance, so that $G_{a}^{B}=(0.88825 / u) \AA^{-2}=5.349 \cdot 10^{46}(\mathrm{~N}$ $\mathrm{m})^{-1} \mathrm{~s}^{-2}$. For the band splitting of the wavenumbers we thus obtain

$$
\begin{aligned}
\Delta \bar{\nu} & =\bar{\nu}_{1}-\bar{\nu}_{2} \\
& =\left(\frac{1}{2 \pi c}\right)^{2} \cdot \frac{G_{a}^{B} \cdot\left\{2 f_{3,2^{\prime}}-2\left(f_{3,6}+f_{3,6^{\prime}}\right)-4 f_{3,5}\right\}}{\bar{\nu}_{1}+\bar{\nu}_{2}}
\end{aligned}
$$

Inserting the values of Table IV] i. e. $\left(2 f_{3,2^{\prime}}-2\left(f_{3,6}+\right.\right.$ $\left.\left.f_{3,6^{\prime}}\right)-4 f_{3,5}\right)=15.784 \cdot 10^{-21} \mathrm{Nm}$ for confined $\mathrm{C}_{16} \mathrm{H}_{33} \mathrm{OH}$, as well as the respective wave numbers, that we take from Fig. 11] at low temperatures (labels 1a and 1b: $\bar{\nu}_{1}+\bar{\nu}_{2} \simeq$ $2 \cdot 1467001 / \mathrm{m})$ we get $\Delta \bar{\nu}=810 \mathrm{~m}^{-1} \equiv 8.1 \mathrm{~cm}^{-1}$ for confined $\mathrm{C}_{16} \mathrm{H}_{33} \mathrm{OH}$. 